Pacific Journal of Mathematics

THE POISSON FLOW ASSOCIATED WITH A MEASURE 


\title{
THE POISSON FLOW ASSOCIATED WITH A MEASURE
}

\section{Douglas Paul Dokken and Robert Ellis}

\begin{abstract}
This paper is devoted to the study of harmonic functions on groups. The approach is via a detailed study of the Poisson flow associated with a Borel probability measure $\mu$ on a locally compact group $T$. Again the basic idea is that though many results associated with the study of harmonic functions on groups are couched in probabilistic terms and proved using methods of probability theory, they really belong in the domain of topological dynamics. The major results include a proof that a solvable connected Lie group admits only constants as harmonic functions for a spread out measure $\mu$ with $\mu(A)=\mu\left(A^{-1}\right)$ for all Borel sets $A$, and a new non-geometric proof of a fundamental result of Furstenberg's on semi-simple Lie groups.
\end{abstract}

0. Introduction. The technical aspects of the paper depend on the methods and results developed in [E] and [D]. For the sake of completeness these are summarized in $\S 1$.

In $\S 2$ another approach to the Poisson flow is given. Let $\mathscr{R}$ be the algebra of right uniformly continuous functions on $T,|\mathscr{R}|$ its Gelfand space and $\mathscr{L}(\mu)$ the set of idempotent measures $\nu$ on $|\mathscr{R}|$ stationary with respect to $\mu$ and having the same harmonic functions. If the support of $\mu$ is all of $T$, the support $S$ of $\nu$ is a subflow of $|\mathscr{R}|$. The main result of this section is that in this case there exists $\nu \in \mathscr{L}(\mu)$ such that the restriction $R: \mathscr{R} \rightarrow C(S)$ maps the set $\mathscr{H}_{\mu}$ of $\mu$-harmonic functions isometrically onto a uniformly closed $T$-invariant subalgebra $\mathscr{K}_{\mu}$ of $C(S)$. The Poisson flow $(B, T)$ is just the Gelfand space of $\mathscr{H}_{\mu}$. This has several implications, among them that $\omega p \in B(p \in S)$ where $\omega$ is the measure on $B$ induced by $\nu$. Moreover the algebra of the flow $(\overline{\nu T}, T)$ is isomorphic to the subalgebra of $\mathscr{R}$ generated by $\mathscr{K}_{\mu}$ and $(B, T)$ is the subflow of $(\overline{\nu T}, T)$ given by $B=\{\nu p \mid p \in S\}$. In this paper the results of this rather technical section are used only in $\S 5$.

Another aim of this paper is to obtain conditions under which a subgroup $K$ of $T$ will act transitively on $B$. The particular case $K=$ $\{e\}$ says that the constants are the only $\mu$-harmonic functions.

Sections 3 and 4 are devoted to this issue. In the former, conditions are studied which suffice to guarantee that a particular element of $T$ 
will act trivially on $B$, and the latter to the study of what happens when the only $K$-invariant harmonic functions are constants. One result along these lines: let $T$ be connected, and $K$ a compact normal subgroup of $T$ such that there are no $K$-invariant harmonic functions other than the constants; then there are no non-constant $\mu$-harmonic functions.

These general results are applied to two specific cases in $\S \S 5$ and 6 .

In $\S 5$ the following result is proved: let $T$ be a solvable connected Lie group, $\mu$ a spread out measure on $T$ such that the closed subgroup generated by the support of $\mu$ is all of $T$ and $\mu(A)=\mu\left(A^{-1}\right)$ for all Borel subsets $A$ of $T$. Then all $\mu$-harmonic functions are constant [B.R.].

Finally $\S 6$ is devoted to a proof of Furstenberg's result on semisimple Lie groups with finite center and no compact factors using the methods developed in $\S \S 3$ and 4. (If $T=K A N$ is an Iwasawa decomposition of such a group, then $K$ acts transitively on the Poisson space of $\mu$ for every supported spread out measure $\mu$ on $T$.)

1.0. Basic background. In this section we introduce the notation and summarize some basic results to be used throughout the rest of the paper. Primary references are $[\mathbf{E}]$ and $[\mathbf{D}]$.

1.1. By a flow we shall mean a transformation group $(X, T)$ with compact Hausdorff phase space $X$ and locally compact phase group $T$. We shall suppress the " $T$ " and denote the flow by $X$.

A pointed flow $\left(X, x_{0}\right)$ is a flow $X$ together with a point $x_{0} \in X$ and $\overline{x_{0} T}=X$. The theory of such flows is the subject of [E] where it is shown that they are all homomorphic images of a universal pointed flow $(\beta T, e)$. (Here $\beta T$ is the Stone-Čech compactification of $T$ with the discrete topology and $e$ is the identity of $T$.)

Let $\left(X, x_{0}\right)$ be a pointed flow. Then there exists a unique epimorphism $\pi: \beta T \rightarrow X$ with $\pi(e)=x_{0}$. The adjoint, $\pi^{*}$ of $\pi$ is a monomorphism of $C(X)$ into $C(\beta T)$ and its image denoted by $\left(X, x_{0}\right)$ is a $T$ subalgebra of $C(\beta T)$; i.e. a uniformly closed $T$-invariant subalgebra of $C(\beta T)$.

Conversely, let $\mathscr{A}$ be a $T$-subalgebra of $C(\beta T)$ and set $x \equiv y(\mathscr{A})$ if $f(x)=f(y)(f \in \mathscr{A})$. Then $(\mathscr{A})$ is a closed $T$-invariant equivalence relation on $\beta T$ and so induces an action of $T$ on the quotient space $\beta T /(\mathscr{A})$ which we denote $|\mathscr{A}|$. The flow $|\mathscr{A}|$ is pointed by $[e]$, the equivalence class to which $e$ belongs. 
The foregoing shows that one can study either the class of pointed flows or the collection of $T$-subalgebras of $C(\beta T)$. We adopt the latter point of view.

One advantage of doing so is that $C(\beta T)$ may be identified with $\mathscr{B}(T)$, the set of bounded real-valued functions on $T$. Thus if $f \in$ $\mathscr{B}(T)$, it has a unique continuous extension $\bar{f}$ to $\beta T$. (Recall that $T$ may be viewed as a subset of $\beta T$.) On the other hand if $g \in C(\beta T)$, then its restriction $f$ to $T$ is in $\mathscr{B}(T)$ and $\bar{f}=g$. In general we shall not distinguish notationally between an element of $\mathscr{B}(T)$ and its continuous extension to $\beta T$.

So far no mention has been made of the topology $\mathscr{T}$ on $T$. However, it is a simple matter to take this into account; one merely requires that all the $T$-subalgebras $\mathscr{A}$ considered be contained in $\mathscr{R}=\{f \in$ $\mathscr{B}(T) \mid f$ is right uniformly continuous $\}$. This will guarantee that the map $(x, t) \rightarrow x t:|\mathscr{A}| \times T \rightarrow|\mathscr{A}|$ is continuous when $T$ is provided with the topology $\mathscr{T}$.

Of course when $\mathscr{T}$ is the discrete topology on $T, \mathscr{R}=C(\beta T)$. Moreover in general $|\mathscr{R}|$ has all the algebraic properties of $\beta T$ and may replace it in all discussions involving flows $X$ where the map $(x, t) \rightarrow x t: X \times(T, \mathscr{T}) \rightarrow X$ is continuous.

1.2. Another advantage of the algebraic approach is that it allows one to incorporate the study of measures on flows into this scheme.

Thus let $\mu$ be a positive linear functional on $\mathscr{B}(T)$ with $\mu(1)=1$. Then under the identification of $\mathscr{B}(T)$ with $C(\beta T), \mu$ may be viewed as a positive linear functional on $C(\beta T)$ and all of its subalgebras. Consequently $\mu$ induces $\mu_{\mathscr{A}} \in \mathscr{M}(|\mathscr{A}|) \equiv \mathscr{M}(\mathscr{A})$, the set of Borel probability measures on $|\mathscr{A}|$ for every $T$-subalgebra $\mathscr{A}$ of $C(\beta T)$.

In particular if $\mu \in \mathscr{M}(T)$, the set of Borel probability measures on $T$, it is defined on bor $(T)$, the set of bounded Borel functions on $T$. The latter is a $T$-invariant uniformly closed subalgebra of $\mathscr{B}(T)$ and so by the Hahn-Banach theorem $\mu$ may be extended to an element $\bar{\mu}$ of $\mathscr{M}=\mathscr{M}(\beta T)$. Of course there are many choices for $\bar{\mu}$ but which one is made is irrelevant for our analysis and so it will also be denoted by $\mu$.

Now let $X$ be a flow, $\nu \in \mathscr{M}(X)$ the set of probability measures on $X$ and $\mu \in \mathscr{M}=\mathscr{M}(B T)$. For $f \in C(X), t \in T$ set

$$
f \nu(t)=\langle t f, \nu\rangle \equiv \int_{X} f(x t) d \nu(x)
$$


Then $f \nu \in \mathscr{B}(T) \equiv C(\beta T)$ whence $\langle f \nu, \mu\rangle=\int_{\beta T} \bar{f} \nu(p) d \mu(p)$ is defined.

The map $f \rightarrow\langle f \nu, \mu\rangle: C(X) \rightarrow \mathbb{R}$ defines a positive linear functional $\nu \mu$ on $C(X)$ with $\nu \mu(1)=1$. Thus $\nu \mu \in \mathscr{M}(X)$.

At this point a word of caution is in order. Let $f$ be a bounded Borel function on $X$. Then $f \nu(t)=\int_{X} f(x t) d \nu(X)$ again defines an element $f \nu$ of $\mathscr{B}(T)$ and so $\int_{\beta T} \overline{f \nu}(p) d \mu(p)$ is defined. However in general this is not equal to $\int_{X} f(x) d(\nu \mu)(x)$. For example: let $f$ be a bounded lower semicontinuous function on $X$. Then

$$
\int_{X} f(x) d(\nu \mu)(x) \leq \int_{\beta T} \overline{f \nu}(p) d \mu(p),
$$

but the two need not be equal.

Now it isn't hard to see that $f \in \operatorname{bor}(X)$ implies that $f \nu \in \operatorname{bor}(T)$. Hence if $\mu \in \mathscr{M}(T)$,

$$
\int_{\beta T} \overline{f \nu}(p) d \mu(p)=\int_{T} f \nu(t) d \mu(t)=\int_{X} f(x) d(\nu \mu)(x) .
$$

Thus in this case $\nu \mu$ is the usual convolution of the measures $\nu$ and $\mu$.

The map $(\nu, \mu) \stackrel{\pi}{\rightarrow} \nu \mu: \mathscr{M}(X) \times \mathscr{M} \rightarrow \mathscr{M}(X)$ defines an action of $\mathscr{M}$ on $\mathscr{M}(X)$ such that the map $\mu \rightarrow \nu \mu: \mathscr{M} \rightarrow \mathscr{M}(X)$ is continuous for all $\nu \in \mathscr{M}(X)$ and the map $\nu \rightarrow \nu \mu: \mathscr{M}(X) \rightarrow \mathscr{M}(X)$ is continuous for all $\mu \in \mathscr{M}(T)$ [2.1.12 of D].

Again when $(X,(T, \mathscr{T}))$ is a flow, $\nu \in \mathscr{M}(X), f \nu \in \mathscr{R}(f \in C(X))$, and $\mathscr{M}$ may be replaced by $\mathscr{M}(\mathscr{R})$ in the preceding discussion.

We shall identify the elements of a compact Hausdorff space $X$ with the subset $\left\{\delta_{y} \mid y \in X\right\} \subset \mathscr{M}(X)$ where $\delta_{y}$ is the Dirac measure at $y$. When this is done the map $\pi$ is seen to be an extension of the action $(x, p) \rightarrow x p: X \times \beta T \rightarrow X$ of $\beta T$ on $X$.

For a detailed discussion of these remarks see [D].

1.3. Notation. Let $\gamma \in \mathscr{M}(\mathscr{R})$. Then $Q_{\gamma} \equiv \overline{\operatorname{cnv}}(\gamma T)$, the closed convex hull of $\gamma T, B_{\gamma} \equiv \overline{\operatorname{ex}}\left(Q_{\gamma}\right)$ the closure of the extreme points of $Q_{\gamma}$, and $\omega_{\gamma} \in \mathscr{M}\left(B_{\gamma}\right)$ with barycenter $b\left(\omega_{\gamma}\right)=\gamma$. The support of $\gamma$ will be denoted $S_{\gamma}$.

Since $(\overline{\gamma T}, \gamma)$ is a flow when $T$ is given the topology $\mathscr{T}$, al $(\overline{\gamma T}, \gamma) \subset$ $\mathscr{R}$ whence there exists a canonical map $\kappa_{\gamma}:|\mathscr{R}| \rightarrow \overline{\gamma T}$ with $\kappa_{\gamma}([e])=\gamma$.

Finally $\mathscr{H}_{\gamma} \equiv\{f \in \mathscr{R} \mid f \gamma=f\}$, the set of harmonic functions with respect to $\gamma$.

When it is clear which measure is being discussed and there is no chance of confusion, the various subscripts involved will be dropped. 
1.4. Definition. Let $\mu \in \mathscr{M}(T), \gamma \in \mathscr{M}(\mathscr{R})$. Then $\gamma$ is stationary with respect to $\mu$ if $\gamma \mu=\gamma$. The set of idempotent, stationary measures $\gamma$ with $\mathscr{H}_{\gamma}=\mathscr{H}_{\mu}$ will be denoted $\mathscr{L}(\mu)$. Thus $\gamma \in \mathscr{L}(\mu)$ if and only if $\gamma^{2}=\gamma, \gamma \mu=\gamma$, and $\mathscr{H}_{\gamma}=\mathscr{H}_{\mu}$.

1.5. Remarks 1. Let $\mu \in \mathscr{M}(T)$. Then there exists $\nu \in \overline{\operatorname{cnv}}\left\{\mu^{n} \mid n=\right.$ $1,2, \ldots\}$ with $\nu \in \mathscr{L}(\mu)$.

2. Let $\mu \in \mathscr{M}(T), \gamma \in \mathscr{L}(\mu)$. Then: (a) $\left(B_{\gamma}, T\right)$ is the Poisson flow associated with $\mu$ [F1] [D]. (b) The barycenter map $b: \mathscr{M}\left(B_{\gamma}\right) \rightarrow Q_{\gamma}$ is bijective. (c) $\omega_{\gamma} \mu=\omega_{\gamma}$. (d) $B_{\gamma}$ is a $T$-invariant subset of $Q_{\gamma}$. (e) If $\operatorname{supp} \omega_{\gamma}$ is $T$-invariant, then $B_{\gamma}=\operatorname{supp} \omega_{\gamma}$.

The proof of the following is straightforward and will be omitted.

1.6. Proposition. Let $\nu, \lambda \in \mathscr{L}(\mu)$. Then (i) $\nu \lambda=\nu$ and $\lambda \nu=\lambda$, (ii) the maps $L_{\nu}: \overline{\lambda T} \rightarrow \overline{\nu T}$ and $L_{\lambda}: \overline{\nu T} \rightarrow \overline{\lambda T}$ such that $L_{\nu}(p)=\nu p$ $(p \in \overline{\lambda T})$ and $L_{\lambda}(q)=\lambda q(q \in \overline{\nu T})$ are isomorphisms which are inverse to one another, (iii) $\omega_{\lambda} \circ L_{\nu}^{-1}=\omega_{\nu}$ and $\omega_{\nu} \circ L_{\lambda}^{-1}=\omega_{\lambda}$, (iv) $L_{\nu} \circ \kappa_{\lambda}=\kappa_{\nu}$, and $L_{\lambda} \circ \kappa_{\nu}=\kappa_{\lambda}$.

Thus the flows $\{\overline{\nu T} \mid \nu \in \mathscr{L}(\mu)\}$ are canonically isomorphic. The next proposition shows that the algebras $C(\overline{\nu T})$ are all canonically isomorphic to the uniformly closed subalgebra generated by $\mathscr{H}_{\mu}$.

1.7. Proposition. Let $\kappa:|\mathscr{R}| \rightarrow \overline{\nu T}$ be the canonical map. Then $\kappa^{*}(C(\overline{\nu T}))=\operatorname{al}\left(\mathscr{H}_{\mu}\right)$, the uniformly closed subalgebra generated by the $\mu$-harmonic functions.

Proof. Let $f \in \mathscr{H}_{\mu}$ and $a(f)$ its affine extension to $\mathscr{M}(\mathscr{R})$. Then $g=\left.a(f)\right|_{\overline{\nu T}} \in C(\overline{\nu T})$ and

$$
\begin{aligned}
\left(\kappa_{\nu}^{*} g\right)(p) & =g(\kappa(p))=g(\nu p)=\langle f, \nu p\rangle=\left\langle f \nu, \delta_{p}\right\rangle \\
& =\left\langle f, \delta_{p}\right\rangle=f(p) \quad(p \in|\mathscr{R}|) .
\end{aligned}
$$

Hence $\operatorname{im} \kappa^{*} \supset \mathscr{H}_{\mu}$ and so im $\kappa^{*} \supset$ al $\left(\mathscr{H}_{\mu}\right)$.

On the other hand let $p, q \in|\mathscr{R}|$ be such that $f(p)=f(q)\left(f \in \mathscr{H}_{\mu}\right)$ and suppose that $h(\nu p)=\left(\kappa^{*} h\right)(p) \neq\left(\kappa^{*} h\right)(q)=h(\nu q)$ for some $h \in C(\overline{\nu T})$. Since the elements of $C(\overline{\nu T})$ of the form $\left.a(f)\right|_{\overline{\nu T}}, f \in \mathscr{R}$, separate points of $\overline{\nu T}$ there exists $f \in C(\overline{\nu T})$ with $f \nu(p)=\langle f, \nu p\rangle \neq$ $\langle f, \nu q\rangle=f \nu(q)$. But this contradicts the original assumption since $f \nu \in \mathscr{H}_{\mu}$. The proof is completed.

1.8. Corollary. Let $(X, T, \omega)$ be a flow such that $\omega \mu=\omega$, and $\nu \in \mathscr{L}(\mu)$. Then there exists a homomorphism $\varphi: \overline{\nu T} \rightarrow \overline{\omega T} \subset \mathscr{M}(X)$ with $\varphi(\nu)=\omega$ (compare 4.4 of $[\mathbf{G}])$. 
Proof. Let $f \in C(X)$. Then $f \omega \mu=f \omega$ implies that $f \omega \in \mathscr{H}_{\mu}=$ $\mathscr{H}_{\nu}$. Hence $f \omega \nu=f \omega$. Thus $\omega \nu=\omega$ and the canonical map $p \rightarrow$ $\omega p:|\mathscr{R}| \rightarrow \overline{\omega T}$ factors through $\overline{\nu T}$.

2.0. The Poisson flow associated with a measure $\mu \in \mathscr{M}(T)$. Some more results concerning the Poisson flow $\left(B_{\gamma}, T, \omega_{\gamma}\right)$ are obtained under the additional assumption that $\operatorname{supp} \mu=T$. Then it is immediate that $\kappa_{\gamma}\left(S_{\gamma}\right) \supset B_{\gamma}$. It is shown that there exists $\lambda \in \mathscr{L}(\mu)$ with $\kappa_{\lambda}\left(S_{\lambda}\right)=B_{\lambda}$. In this case the restriction $\left.f \rightarrow f\right|_{S_{\lambda}}: \mathscr{R} \rightarrow C\left(S_{\lambda}\right)$ maps $\mathscr{H}_{\lambda}$ isometrically onto a uniformly closed $T$-invariant subalgebra of $C\left(S_{\lambda}\right)$.

2.1. LEMMA. Let $X$ be a flow and $\eta \in \mathscr{M}(\mathscr{R})$ be such that the map $\gamma \rightarrow \gamma \eta: \mathscr{M}(X) \rightarrow \mathscr{M}(X)$ is continuous. Then $\langle f, \gamma \eta\rangle=\int_{X}\left\langle f, \delta_{x} \eta\right\rangle d \gamma(x)$ $(\gamma \in \mathscr{M}(X), f \in C(X))$.

Proof. Let $K$ be the set of measures on $X$ for which 2.1 is valid. Then clearly $K$ is convex and the assumption on $\eta$ implies that it is closed.

Now if $y \in X, \int_{X}\left\langle f, \delta_{x} \eta\right\rangle d \delta_{y}(x)=\left\langle f, \delta_{y} \eta\right\rangle$ so that $X \subset K$. Hence $K=\mathscr{M}(X)$.

2.2. Proposition. Let $X$ be a flow and $\eta \in \mathscr{M}(\mathscr{R})$ be such that the map $\gamma \rightarrow \gamma \eta: \mathscr{M}(X) \rightarrow \mathscr{M}(X)$ is continuous. Then ( $\overline{\text { supp }}) \overline{\operatorname{supp} \eta}=$ $\operatorname{supp} \gamma \eta(\gamma \in \mathscr{M}(X))$.

Proof. Let $\gamma \in \mathscr{M}(X), u \in \operatorname{supp} \gamma, p \in \operatorname{supp} \eta$ and $f \in C(X)$ with $f \geq 0$ and $f(u p)>0$. Then the map $f u:|\mathscr{R}| \rightarrow \mathbb{R}$ such that $f u(q)=$ $f(u q)(q \in|\mathscr{R}|)$ is continuous and positive. Consequently $\left\langle f, \delta_{u} \eta\right\rangle=$ $\left\langle f \delta_{u}, \eta\right\rangle=\langle f u, \eta\rangle \geq a>0$ since $p \in \operatorname{supp} \eta$. This implies by 2.1 that $\langle f, \gamma \eta\rangle=\int_{X}\left\langle f, \delta_{x} \eta\right\rangle d \gamma(x)>0$ since $u \in \operatorname{supp} \gamma$, whence $u p \in \operatorname{supp} \gamma \eta$. Thus $(\operatorname{supp} \gamma)(\operatorname{supp} \eta) \subset \operatorname{supp} \gamma \eta$ and so $(\overline{\operatorname{supp} \gamma})(\overline{\operatorname{supp} \eta}) \subset \overline{\operatorname{supp} \gamma \eta}=$ supp $\gamma \eta$.

Now assume $u \notin \overline{(\operatorname{supp} \gamma)(\operatorname{supp} \eta)}$. Then there exists $f \in C(X)$ with $0 \leq f \leq 1, f(u)=1$, and $\operatorname{supp} f$ contained in the complement of $(\operatorname{supp} \gamma)(\operatorname{supp} \eta)$.

Then $0=f(x p)=\left\langle f, \delta_{x} p\right\rangle(x \in \operatorname{supp} \gamma, p \in \operatorname{supp} \eta)$ whence $\left\langle f, \delta_{x} \eta\right\rangle=0(x \in \operatorname{supp} x)$. Thus

$$
\langle f, \gamma \eta\rangle=\int_{X}\left\langle f, \delta_{x} \eta\right\rangle d \gamma(x)=\int_{\operatorname{supp} \gamma}\left\langle f, \delta_{x} \eta\right\rangle d \gamma(x)=0
$$

whence $u \notin \operatorname{supp} \gamma \eta$. The proof is completed. 
2.3. Corollary. Let $\mu \in \mathscr{M}(T)$ with $\operatorname{supp} \mu=T$, and $\gamma \in \mathscr{L}(\mu)$. Then (a) $S_{\gamma}$ is $T$-invariant, (b) supp $\omega_{\gamma}$ is $T$-invariant and (c) $\operatorname{supp} \omega_{\gamma}$ $=B_{\gamma}$.

Proof. Since $\gamma \mu=\gamma$ and $\omega_{\gamma} \mu=\omega_{\gamma}$, (a) and (b) follow from 2.2.

The image of $\overline{\operatorname{cnv}}\left(\operatorname{supp} \omega_{\gamma}\right)$ under the barycenter map is a closed, convex, $T$-invariant subset of $Q_{\gamma}$ which contains $\gamma$. Hence

$$
b\left(\overline{\operatorname{cnv}}\left(\operatorname{supp} \omega_{\gamma}\right)\right)=Q_{\gamma},
$$

whence supp $\omega_{\gamma}=\operatorname{ex}\left(\overline{\operatorname{cnv}} \operatorname{supp} \omega_{\gamma}\right)=b\left(\operatorname{ex} \overline{\operatorname{cnv}} \operatorname{supp} \omega_{\gamma}\right)=\operatorname{ex} Q_{\gamma}=B_{\gamma}$. (Recall that $b$ is bijective.)

2.4. Proposition. Let $\mu \in \mathscr{M}(T)$, $\operatorname{supp} \mu=T$, and $\gamma \in \mathscr{L}(\mu)$. Then $\kappa_{\gamma}\left(S_{\gamma}\right) \supset B_{\gamma}$.

Proof. Let $\bar{\kappa}$ be the canonical extension of $\kappa_{\gamma}$ to a map from $\mathscr{M}(\mathscr{R})$ to $\overline{\operatorname{cnv}}(\gamma T) \subset \mathscr{M}(\mathscr{R})$. Then $\bar{\kappa}\left(\overline{\operatorname{cnv}} S_{\gamma}\right)$ is a closed convex $T$-invariant subset of $\overline{\operatorname{cnv}}(\gamma T)$ containing $\gamma$. Hence $\bar{\kappa}\left(\overline{\operatorname{cnv}} S_{\gamma}\right)=\overline{\operatorname{cnv}}(\gamma T)$ whence

$$
\kappa\left(S_{\gamma}\right)=\bar{\kappa}\left(S_{\gamma}\right)=\bar{\kappa}\left(\operatorname{ex}\left(\overline{\operatorname{cnv}} S_{\gamma}\right)\right) \supset \operatorname{ex}(\overline{\operatorname{cnv}}(\gamma T))=B_{\gamma} .
$$

Our goal now is to find $\gamma \in \mathscr{L}(\mu)$ with $\kappa_{\gamma}\left(S_{\gamma}\right)=B_{\gamma}$.

2.5. Notation. For the rest of this section the following notation will be in force: $\mu \in \mathscr{M}(T)$, supp $\mu=T, \nu \in \mathscr{L}(\mu), S=S_{\nu}, \kappa=\kappa_{\nu}$, $B=B_{\nu}, \omega=\Omega_{\nu}$ and $L=\kappa^{-1}(B) \cap S$. Notice that $L$ is a closed invariant subset of $S$.

2.6. Lemma. Let $\lambda \in \mathscr{M}(\mathscr{R}), \operatorname{supp} \lambda \subset L$, and $\lambda \kappa^{-1}=\omega$. Then $\nu \lambda=\nu$.

Proof. Let $f \in \mathscr{R}, p, q \in L$ with $\kappa(p)=\kappa(q)$ i.e. $\nu p=\nu q$. Then $f \nu(p)=\langle f, \nu p\rangle=\langle f, \nu q\rangle=f \nu(q)$ whence $f \nu=\varphi \circ \kappa$ for some $\varphi \in C(B)$.

Now $\langle f, \nu \lambda\rangle=\langle f \nu, \lambda\rangle=\langle\varphi \circ \kappa, \lambda\rangle=\left\langle\varphi, \lambda \kappa^{-1}\right\rangle=\langle\varphi, \omega\rangle$.

On the other hand let $a(f)$ be the affine extension of $f$ to $\mathscr{M}(\mathscr{R})$ and let $b=\nu p \in B$. Then $a(f)(b)=\langle f, \nu p\rangle=f \nu(p)=\varphi(\kappa(p))=\varphi(b)$ whence $\left.a(f)\right|_{B}=\varphi$. Finally $\langle f, \nu\rangle=a(f)(\nu)=\left\langle\left. a(f)\right|_{B}, \omega\right\rangle=\langle\varphi, \omega\rangle=$ $\langle f, \nu \lambda\rangle$. 
2.7. Lemma. There exists $\lambda^{2}=\lambda \in \mathscr{M}(\mathscr{R})$ such that $\nu \lambda=\nu$, $\operatorname{supp} \lambda \subset L$ and $\lambda \cdot \kappa^{-1}=\omega$.

Proof. Let $K=\{\lambda \in \mathscr{M}(\mathscr{R}) \mid \nu \lambda=\nu, \operatorname{supp} \lambda \subset L\}$.

The Hahn-Banach theorem and 2.6 imply that $K \neq \varnothing$.

Now let $\lambda, \rho \in K$. Then $\nu \lambda \rho=\nu \rho=\nu$. Moreover $\lambda \in \overline{\operatorname{cnv}} \operatorname{supp} \lambda \subset$ $\overline{\mathrm{cnv}} L$ and $\rho \in \overline{\mathrm{cnv}} \operatorname{supp} \rho \subset \overline{\mathrm{cnv}} L$ imply that $\lambda \rho \in(\overline{\mathrm{cnv}} L)(\overline{\mathrm{cnv}} L) \subset$ $\overline{\mathrm{cnv}} L$ (recall $L$ is $T$-invariant), whence $\operatorname{supp} \lambda \rho \subset L$.

Thus $K$ is a non-empty semigroup. Since it is clearly closed, there exists $\lambda \in K$ with $\lambda^{2}=\lambda$.

Finally let $\gamma=\lambda \cdot \kappa^{-1}, f \in \mathscr{R}$. Then

$$
\begin{aligned}
\left\langle\left. a(f)\right|_{B}, \gamma\right\rangle & =\langle a(f) \cdot \kappa, \lambda)=\langle f \nu, \lambda\rangle=\langle f, \nu \lambda\rangle \\
& =\langle f, \nu\rangle=a(f)(\nu) .
\end{aligned}
$$

Hence $b(\gamma)=\nu=b(\omega)$ and so $\gamma=\omega$ since $b$ is one-one [D, 3.1.8].

2.8. Proposition. There exists $\lambda \in \mathscr{L}(\mu)$ with $\kappa_{\lambda}\left(S_{\lambda}\right)=B_{\lambda}$.

Proof. Let $\rho^{2}=\rho \in \mathscr{M}(\mathscr{R})$ with $\nu \rho=\nu$ and $\operatorname{supp} \rho \subset L$. Such exists by 2.7 .

Set $\lambda=\rho \nu$. Then $\lambda^{2}=\rho \nu \rho \nu=\rho \nu=\lambda, \lambda \nu=\rho \nu^{2}=\rho \nu=\lambda$ and $\nu \lambda=\nu \rho \nu=\nu^{2}=\nu$. Consequently $\mathscr{H}_{\lambda}=\mathscr{H}_{\nu}=\mathscr{H}_{\mu}$ and so $\lambda \in \mathscr{L}(\mu)$.

Since $L$ is $T$-invariant and $\operatorname{supp} \rho \subset L, \operatorname{supp} \lambda \subset L$.

Now $L_{\lambda}: \overline{\nu T} \rightarrow \overline{\lambda T}$ is an isomorphism (1.6) whence $L_{\lambda}\left(B_{\nu}\right)=B_{\lambda}$. Hence $\kappa_{\lambda}\left(S_{\lambda}\right) \subset \kappa_{\lambda}(L)=L_{\lambda} \kappa_{\nu}(L)=L_{\lambda}\left(B_{\nu}\right)=B_{\lambda}$. The proof is complete. (2.4).

2.9. Notation. For the rest of this section $\lambda$ will denote a measure guaranteed by 2.8. Thus $\lambda \in \mathscr{L}(\mu)$ with $\nu \lambda=\nu, \lambda \nu=\lambda, S_{\lambda} \subset S_{\nu}$ and $\kappa_{\lambda}\left(S_{\lambda}\right)=B_{\lambda}$. The restriction of $\kappa_{\lambda}$ to $S_{\lambda}$ will be denoted by $r_{\lambda}$.

2.10. Lemma. Let $\gamma \in \mathscr{L}(\mu), f, g \in \mathscr{H}_{\mu}$ with $f(p)=g(p)\left(p \in S_{\gamma}\right)$. Then $f=g$.

Proof. Let $t \in T$. Then

$$
\begin{aligned}
f(t) & =f \gamma(t)=\int_{S_{\gamma}} f(x t) d \gamma(x) \\
& =\int_{S_{\gamma}} g(x t) d \gamma(x)=g \gamma(t)=g(t) .
\end{aligned}
$$

(Recall that $S_{\gamma}$ is invariant.) 
2.11. Definition. Let $\gamma \in \mathscr{L}(\mu)$ and $f \in C\left(S_{\gamma}\right)$. Then $f$ is harmonic on $S_{\gamma}$ if there exists $g \in \mathscr{H}_{\mu}$ with $\left.g\right|_{S_{\gamma}}=f$. (Notice that by 2.10 if such a $g$ exists, it is unique.) The set of harmonic functions on $S_{\gamma}$ will be denoted by $\mathscr{H}_{\text {. }}$.

2.12. Proposition. Let $\varphi: C\left(B_{\lambda}\right) \rightarrow \mathscr{R}$ be the map such that $\varphi(f)=$ $f \omega_{\lambda}\left(f \in C\left(B_{\lambda}\right)\right)$ and $R: \mathscr{R} \rightarrow C\left(S_{\lambda}\right)$ the restriction map. Then (i) $R \circ \varphi=r_{\lambda}^{*}$, (ii) $\mathscr{K}_{\lambda}=\mathrm{im} r_{\lambda}^{*}$, (iii) $r_{\lambda}^{*}$ is an isometry, (iv) $R$ restricted to $\mathscr{H}_{\mu}$ is an isometry into $\mathscr{K}_{\lambda}$.

Proof. (i) Let $f \in C\left(B_{\lambda}\right), g=\varphi(f), a(g)$ the affine extension of $g$ to $\mathscr{M}(\mathscr{R})$ and $p \in S_{\lambda}$. Then $\kappa(p) \in B_{\lambda}$ and by [D, 3.1.6],

$$
\begin{aligned}
f(\kappa(p)) & =a(g)(\kappa(p))=\langle g, \lambda p\rangle=\left\langle f \lambda, \lambda \delta_{p}\right\rangle=\left\langle f \lambda^{2}, \delta_{p}\right\rangle \\
& =\left\langle f \lambda, \delta_{p}\right\rangle=\left\langle g, \delta_{p}\right\rangle=g(p) .
\end{aligned}
$$

(ii) By $[\mathbf{D}, 2.1 .22] \varphi$ is an isometry onto $\mathscr{H}_{\mu}$ whence im $r_{\lambda}^{*}=R\left(\mathscr{H}_{\mu}\right)=$ $\mathscr{H}_{\lambda}$ by 2.12 .

(iii) This follows from the fact that $\kappa_{\lambda}\left(S_{\lambda}\right)=B_{\lambda}$.

(iv) Let $f \in \mathscr{H}_{\mu}$. Then by [D, 2.1.22] there exists $g \in C\left(B_{\lambda}\right)$ with $\varphi(g)=f$ and $\|g\|=\|f\|$. Then $R(f)=R(\varphi(g))=\kappa^{*}(g)$ whence $\|R(f)\|=\left\|\kappa^{*}(g)\right\|=\|g\|=\|f\|$.

2.13. Remark 1. Proposition 2.12 shows that $\mathscr{K}_{\lambda}$ is a uniformly closed $T$-invariant subalgebra of $C\left(S_{\lambda}\right)$ and that $B_{\lambda}$ is obtained from $S_{\lambda}$ by identifying points with respect to the relation $\left\{(x, y) \mid x, y \in S_{\lambda}\right.$, $\left.f(x)=f(y)\left(f \in \mathscr{K}_{\lambda}\right)\right\}$. Thus the harmonic functions on $S_{\lambda}$ are a subalgebra of $C\left(S_{\lambda}\right)$ whereas in general $\mathscr{H}_{\mu}=\mathscr{H}_{\lambda}$ is not a subalgebra of $\mathscr{R}=C(|\mathscr{R}|)$.

2.14. Proposition. Let $R: \mathscr{R} \rightarrow C\left(S_{\lambda}\right)$ be the restriction map. Then $R$ induces a homomorphism $R_{\lambda}$ of $\mathrm{al}\left(\mathscr{H}_{\mu}\right)$ onto $\mathscr{K}_{\lambda}$ and $\mathrm{al}\left(\mathscr{H}_{\mu}\right)=$ $\mathscr{H}_{\mu} \oplus R_{\lambda}^{-1}(0)$.

Proof. By $2.12 \mathscr{K}_{\lambda}$ is a uniformly closed $T$-invariant subalgebra of $C\left(S_{\lambda}\right)$ whence $R$ induces a homomorphism of $R^{-1}\left(\mathscr{K}_{\lambda}\right)$ onto $\mathscr{K}_{\lambda}$. Since $R\left(\mathscr{H}_{\mu}\right)=\mathscr{K}_{\lambda}, \operatorname{al}\left(\mathscr{H}_{\mu}\right) \subset R^{-1}\left(\mathscr{K}_{\lambda}\right)$, and so $R$ induces a homomorphism $R_{\lambda}$ of al $\left(\mathscr{H}_{\mu}\right)$ onto $\mathscr{K}_{\lambda}$.

Now let $f \in \operatorname{al}\left(\mathscr{H}_{\mu}\right)$. Then $R_{\lambda} f \in \mathscr{K}_{\lambda}$ and so there exists $g \in \mathscr{H}_{\mu}$ with $R_{\lambda} f=R_{\lambda} g$. Thus $\operatorname{al}\left(\mathscr{H}_{\mu}\right)=\mathscr{K}_{\lambda}+R_{\lambda}^{-1}(0)$. That the sum is direct now follows from 2.10 . 
2.15. Remarks. 1. Let $\gamma \in \mathscr{L}(\mu), f \in \mathrm{al}\left(\mathscr{H}_{\mu}\right)$, and $a(f)$ the affine map on $C(\overline{\gamma T})$ induced by $f$. Then $a(f)(\gamma t)=\langle f, \gamma t\rangle=(f \gamma)(t)(t \in$ $T)$. Thus $a(f) \circ \kappa_{\gamma}=f \gamma$. This shows that in general the isomorphism $\kappa_{\lambda}^{*}$ of $C(\overline{\gamma T})$ onto al $\left(\mathscr{H}_{\mu}\right)$ takes the affine functions $a(\overline{\gamma T})=\{a(f) \mid f \in$ $\mathscr{R}\}$ onto the harmonic functions $\mathscr{H}_{\mu}$.

2. In the case of $\lambda$ we can say a little more. Thus let $O\left(B_{\lambda}\right)=\{f \in$ $\left.C(\overline{\lambda T}):\left.f\right|_{B_{\lambda}}=0\right\}$. Then it is easy to see that $f \in O\left(B_{\lambda}\right)$ if and only if $R_{\lambda}\left(f \circ \kappa_{\lambda}\right)=0$. Consequently the splitting al $\left(\mathscr{H}_{\mu}\right)=\mathscr{H}_{\mu} \oplus R_{\lambda}^{-1}(0)$ induces the splitting $C(\overline{\lambda T})=a(\overline{\lambda \tau}) \oplus O\left(B_{\lambda}\right)$.

3. Let $g, h \in \mathscr{R}$ with $\left.h\right|_{s_{\lambda}}=0$. Then $a(g+h)=a(g)$. Thus if $f \in C(\overline{\lambda T})$ with $f=a(g)+l$ with $l \in O\left(B_{\lambda}\right)$ then $f=a(g+h)+l$; i.e. even though the decomposition of $f$ in the form $f=a(g)+l$ is unique, the element $g$ of $\mathscr{R}$ is not. One choice for $g$ is $f \circ \kappa_{\lambda}$.

To see this observe that $f \circ \kappa_{\lambda} \in \operatorname{al}\left(\mathscr{H}_{\mu}\right)$ whence by $2.14 f \circ \kappa_{\lambda}=h+u$ where $h \in \mathscr{H}_{\mu}$ and $u \equiv 0$ on $S_{\lambda}$. Then $h=h \lambda=h \lambda+u \lambda=\left(f \circ \kappa_{\lambda}\right) \lambda$. $0 \equiv f \circ \kappa_{\lambda}-\left(f \circ \kappa_{\lambda}\right) \lambda=f \circ \kappa_{\lambda}-a\left(f \circ \kappa_{\lambda}\right) \circ \kappa_{\lambda}$ on $S_{\lambda}$ by Remark 1 above. Consequently $f-a\left(f \circ \kappa_{\lambda}\right) \equiv 0$ on $B_{\lambda}$.

4. Let $f \in C\left(B_{\lambda}\right), g, l \in C(\overline{\lambda T})$ and $h \in \mathscr{R}$ such that $\left.g\right|_{B_{\lambda}}=f$, $l \in O\left(B_{\lambda}\right)$ and $g=a(h)+l$. Then $\left.a(h)\right|_{B_{\lambda}}=\left.g\right|_{B_{\lambda}}=f$. Thus given any continuous function $f$ on the boundary there exists an "harmonic function" $a(h)$ with $\left.a(h)\right|_{B_{\lambda}}=f$.

5. The isomorphism $\kappa_{\lambda}^{*}$ of $C(\overline{\lambda T})$ onto al $\left(\mathscr{H}_{\mu}\right)$ also induces an isomorphism $\hat{\kappa}_{\lambda}$ of $\mathscr{M}\left(\mathrm{al}\left(\mathscr{H}_{\mu}\right)\right)$ onto $\mathscr{M}(\overline{\lambda T})$. Now $\lambda$ may be viewed as a measure on $\operatorname{al}\left(\mathscr{H}_{\mu}\right)$ by restrition and $\omega_{\lambda}$ as a measure on $\overline{\lambda T}$ since $B_{\lambda} \subset \overline{\lambda T}$. It is natural to expect that $\hat{\kappa}_{\lambda}(\lambda)=\omega_{\lambda}$. To see that this is indeed the case let $f \in C(\overline{\lambda T})$. Then $\left\langle f, \omega_{\lambda}\right\rangle=\int_{B_{\lambda}} f d \omega_{\lambda}=\int_{S_{\lambda}} f \circ \kappa_{\lambda} d \lambda=$ $\left\langle f, \hat{\kappa}_{\lambda}(\lambda)\right\rangle$. (Use 2.7.)

The aim of the following is to compare the construction of the Poisson flow above with the one given originally by Furstenberg [F1]. (See also $[\mathbf{A}]$ and $[\mathbf{G}]$.)

2.16. Remarks. 1. The Poisson flow constructed by Furstenberg is obtained by showing: (i) $f \rho=\lim _{n \rightarrow \infty} f \mu^{n}$ exists for all $f \in \operatorname{al}\left(\mathscr{H}_{\mu}\right)$, (ii) $\mathscr{J}=\{f \mid f \rho=0\}$ is an ideal in al $\left(\mathscr{H}_{\mu}\right)$ and (iii) al $\left(\mathscr{H}_{\mu}\right)=\mathscr{H}_{\mu} \oplus \mathscr{J}$. The resulting isomorphism of $\mathscr{H}_{\mu}$ with al $\left(\mathscr{H}_{\mu}\right) / \mathscr{J}$ allows one to define a product $f * g=(f g) \rho$ on $\mathscr{H}_{\mu}$ and a measure $\omega_{\rho}(f)=f(e)\left(f \in \mathscr{H}_{\mu}\right)$ such that $\left(B_{\rho}, T, \omega_{\rho}\right)$ is the Poisson flow associated with $\mu$ where $B_{\rho}$ is the Gelfand space of the Banach algebra $\left(\mathscr{H}_{\mu}, *\right)$. Moreover the map $\varphi_{\rho}: C\left(B_{\rho}\right) \rightarrow\left(\mathscr{H}_{\mu}, *\right)$ such that $\varphi_{\rho}(f)=f \omega_{\rho}\left(f \in C\left(B_{\rho}\right)\right)$ is an algebra isomorphism. 
2. By Proposition $2.12, R_{\lambda}$ is an isometry of $\mathscr{H}_{\mu}$ onto the uniformly closed $T$-invariant subalgebra $\mathscr{K}_{\lambda}$ of $C\left(S_{\lambda}\right)$. Hence the equation $R_{\lambda}(f \Lambda g)=R_{\lambda}(f) R_{\lambda}(g)\left(f, g \in \mathscr{H}_{\mu}\right)$ defines a multiplication $\Lambda$ on $\mathscr{H}_{\mu}$ which makes it into a commutative Banach algebra.

Now let $f, g \in C\left(B_{\lambda}\right)$. Then again by $2.12, R\left(f \omega_{\lambda}\right) R\left(g \omega_{\lambda}\right)=$ $\left(f \circ r_{\lambda}\right)\left(g \circ r_{\lambda}\right)=f g \circ r_{\lambda}=R\left((f g) \omega_{\lambda}\right)$. Hence $f \omega_{\lambda} \Lambda g \omega_{\lambda}=(f g) \omega_{\lambda}$ and so the map $\varphi_{\lambda}: C\left(B_{\lambda}\right) \rightarrow\left(\mathscr{H}_{\mu}, \Lambda\right)$ such that $\varphi_{\lambda}(f)=f \omega_{\lambda}$ is an algebra isomorphism.

3. Since all Poisson flows are isomorphic there exists an isomorphism $\psi: C\left(B_{\rho}\right) \rightarrow C\left(B_{\lambda}\right)$ such that $f \omega_{\lambda}=(f \circ \psi) \omega_{\rho}\left(f \in C\left(B_{\lambda}\right)\right)$.

Let $f, g \in C\left(B_{\lambda}\right)$. Then

$$
\begin{aligned}
f \omega_{\lambda} * g \omega_{\lambda} & =(f \circ \psi) \omega_{\rho} *(g \circ \psi) \omega_{\rho}=((f \circ \psi)(g \circ \psi)) \omega_{\rho} \\
& =((f g) \circ \psi) \omega_{\rho}=(f g) \omega_{\lambda}=f \omega_{\lambda} \Lambda g \omega_{\lambda} .
\end{aligned}
$$

Thus the two multiplications $*, \Lambda$ on $\mathscr{H}_{\mu}$ are the same.

4. Let $f, g \in \mathscr{H}_{\mu}$. Then $f g=h+l$ where $h \in \mathscr{H}_{\mu}$ and $l_{S_{\lambda}} \equiv 0$. (2.17). This implies that $h=h \lambda=(f g) \lambda-l \lambda=(f g) \lambda$ from which it follows that $R_{\lambda}(f g)=R_{\lambda}((f g) \lambda)+R_{\lambda}(l)=R_{\lambda}((f g) \lambda)$. Hence $f \Lambda g=(f g) \lambda$ $\left(f, g \in \mathscr{H}_{\mu}\right)$.

5. Combining 1,3 , and 4 we see that $(f g) \lambda=(f g) \rho\left(f, g \in \mathscr{H}_{\mu}\right)$. Now let $f_{1}, \ldots, f_{n} \in \mathscr{H}_{\mu}$ with $n \geq 3$ and assume that $F \rho=F \lambda$ where $F=f_{1} \cdots f_{n-1}$.

Let $F=h+l$ with $h \in \mathscr{H}_{\mu}$ and $\left.l\right|_{S_{\lambda}} \equiv 0$. The $h=h \lambda=h \lambda+l \lambda=$ $F \lambda=F \rho=h \rho+l \rho=h+l \rho$ whence $l \rho=0$. Since $\rho^{-1}(0)$ is an ideal $\left(l f_{n}\right) \rho=0$. Moreover $\left.l\right|_{S_{\lambda}} \equiv 0$ implies that $\left(l f_{n}\right) \lambda=0$. Consequently $\left(f_{1} \cdots f_{n}\right) \rho=\left(F f_{n}\right) \rho=\left(h f_{n}\right) \rho+\left(l f_{n}\right) \rho=\left(h f_{n}\right) \rho=\left(h f_{n}\right) \lambda$ (by 4 above $)=\left(h f+l f_{n}\right) \lambda=\left(F f_{n}\right) \lambda=\left(f_{1} \cdots f_{n}\right) \lambda$.

Finally since $\rho$ and $\lambda$ are linear, $f \lambda=f \rho=\lim _{n \rightarrow \infty} f \mu^{n}(f \in$ $\left.\operatorname{al}\left(\mathscr{H}_{\mu}\right)\right)$

6. Let $\hat{\kappa}_{\lambda}: \mathscr{M}(|\mathscr{R}|) \rightarrow \mathscr{M}(\overline{\lambda T})$ be the map induced by $\kappa_{\lambda}$, and $\varphi$ : $\mathscr{M}\left(\mathrm{al}\left(\mathscr{H}_{\mu}\right)\right) \rightarrow \mathscr{M}(\overline{\lambda T})$ the isomorphism induced by $\kappa_{\lambda}^{*}: C(\overline{\lambda T}) \rightarrow \operatorname{al}\left(\mathscr{H}_{\mu}\right)$. Then $\hat{\kappa}_{\lambda}=\varphi \circ r$ where $r: \mathscr{M}(\mathscr{R}) \rightarrow \mathscr{M}\left(\mathrm{al}\left(\mathscr{H}_{\mu}\right)\right)$ is the restriction map. Hence $\lim \hat{\kappa}_{\lambda}\left(\mu^{n}\right)=\varphi\left(\lim r\left(\mu^{n}\right)\right)=\varphi(\lambda)=\omega_{\lambda}$ by 5 of 2.15 .

7. In the various papers cited above it is assumed that $T$ is second countable. This is probably not necessary but we don't insist for the following proposition will be used only for such $T$.

2.17. Proposition. Let $T$ be second countable. Then there exists $\gamma \in \mathscr{L}(\mu)$ such that $\gamma \in K=\overline{\operatorname{cnv}}\left\{\mu^{n} \mid n=1, \ldots\right\} \subset \mathscr{M}(\mathscr{R})$ and $\kappa_{\gamma}\left(S_{\gamma}\right)=$ $B_{\gamma}$. 
Proof. Let $\lambda \in \mathscr{L}(\mu)$ with $\kappa_{\lambda}\left(S_{\lambda}\right)=B_{\lambda}$ and $\hat{\kappa}_{\lambda}: \mathscr{M}(|\mathscr{R}|) \rightarrow \mathscr{M}(\overline{\lambda T})$ induced by $\kappa_{\lambda}$. Then by 6 of $2.16, K \cap \hat{\kappa}_{\lambda}^{-1}\left(\omega_{\lambda}\right)$ is nonvacuous. Since it is also compact, convex, and invariant under $\mu$, there exists $\gamma \in$ $K \cap \hat{\kappa}_{\lambda}^{-1}\left(\omega_{\lambda}\right)$ with $\gamma \mu=\gamma$ and thus $\gamma^{2}=\gamma$ since $\gamma \in K$. This implies that $\gamma \in \mathscr{L}(\mu)$ with $\kappa_{\lambda}\left(S_{\gamma}\right)=\operatorname{supp} \omega_{\lambda}=B_{\lambda}$, whence $\kappa_{\gamma}\left(S_{\gamma}\right)=B_{\gamma}$ as in the proof of 2.8 .

3.0. Some basic results. Our aim in most instances is to show that under certain conditions the constants are the only $\mu$-harmonic functions, i.e. $\mathscr{H}_{\mu}=\mathbb{R}$. This is equivalent to showing that the Poisson flow $(B, T)$ is a point or that $T$ acts trivially on $B$.

In this section we prove some basic results which will be applied later to show that $\mathscr{H}_{\mu}=\mathbb{R}$. They are of the form of conditions on $t \in T$ and $x \in B$ which guarantee that $x t=x$.

3.1. Standing notation. Throughout this section $\mu$ will denote a fixed element of $\mathscr{M}(T),(X, T)$ a flow and $\omega \in \mathscr{M}(X)$ with $\omega \mu=\omega$.

3.2. Proposition. Let $\lambda \in \mathscr{M}(T)$ and $f$ a bounded Borel function on $X$. Then (i)

$$
\langle f, \omega \lambda\rangle=\iint_{T \times X} f(x t) d \omega(x) d \lambda(t)=\iint_{X \times T} f(x t) d \lambda(t) d \omega(x)
$$

and (ii)

$$
\omega(A)=\int_{T} \omega\left(A t^{-1}\right) d \mu(t)
$$

for all Borel subsets $A$ of $X$.

Proof. Statement (i) follows from Fubini's theorem and the remarks made in 1.2.

(ii) This follows from (i) with $f=\chi_{A}$.

3.3. Corollary. Let $A$ be a Borel subset of $X$ with $\omega(A)=1$ and $H=\left\{t \in T \mid \omega\left(A t^{-1}\right)=1\right\}$. Then (i) $\mu(H)=1$, (ii) the map $s \rightarrow \omega\left(A s^{-1}\right): T \rightarrow \mathbb{R}$ continuous at $t \in \operatorname{supp} \mu$ implies that $t \in H$.

Proof. (i) Let $H_{n}=\left\{t \mid \omega\left(A t^{-1}\right) \geq 1-\frac{1}{n}\right\}$. Then

$$
\begin{aligned}
1 & =\omega(A)=\int_{H_{n}} \omega\left(A t^{-1}\right) d \mu(t)+\int_{T \backslash H_{n}} \omega\left(A t^{-1}\right) d \mu(t) \\
& \geq \mu\left(H_{n}\right)+\left(1-\frac{1}{n}\right)\left(1-\mu\left(H_{n}\right)\right)=1-\frac{1}{n}+\frac{1}{n} \mu\left(H_{n}\right),
\end{aligned}
$$

whence $\mu\left(H_{n}\right) \geq 1$ and the result follows. 
(ii) Assume $\omega\left(A t^{-1}\right)<a<1$. Then there exists a neighborhood $U$ of $t$ with $\omega\left(A s^{-1}\right)<a(s \in U)$ whence

$$
\begin{aligned}
\omega(A) & =\int_{U} \omega\left(A r^{-1}\right) d \mu(r)+\int_{T \backslash U} \omega\left(A r^{-1}\right) d \mu(r) \\
& <a \mu(U)+1-\mu(U)<1
\end{aligned}
$$

since $\mu(U)>0$. The proof is completed.

3.4. Corollary. Let $X$ be metrizable $x \in X$, and $\left(t_{n}\right)$ a sequence in $T$ such that $\omega t_{n} \rightarrow \delta_{x}$ and $K=\left\{t \in T \mid \omega t t_{n} \rightarrow \delta_{x}\right\}$. Then $\mu(K)=1$.

Proof. There exists a Borel subset $A$ of $X$ with $\omega(A)=1$ and $y t_{n} \rightarrow$ $x(y \in A)\left[\mathbf{A}\right.$, I.2]. Let $t \in T$ with $\omega\left(A t^{-1}\right)=1$. Let $y \in A t^{-1}$. Then $y t \in A$ and $y t t_{n} \rightarrow x$. Thus $y\left(t t_{n}\right) \rightarrow x\left(y \in A t^{-1}\right)$ from which it follows readily that $\omega t t_{n} \rightarrow \delta_{x}$. The result now follows from 3.3.

3.5. Definition. Let $t \in T$. Then $\omega$ is regular at $t$ if there exists $\delta>0$ such that $C$ closed with $\omega(C)>1-\delta$ implies that $C \cap C s \neq \varnothing$ for all $s$ in some neighborhood $U_{C}$ of $t$.

3.6. THEOREM. Let $\left(r_{n}\right),\left(s_{n}\right),\left(t_{n}\right)$ be sequences in $T$ such that $t_{n} s_{n}=$ $r_{n} t_{n}$ for all $n,\left(s_{n}\right),\left(r_{n}\right)$ converge to $s, r \in T$ respectively, $\omega$ regular at $r$, and $x t_{n} \rightarrow b(x \in A)$ with $\omega(A)=1$. Then $b s=b$.

Proof. Let $\delta$ be as in 3.5 guaranteed by the regularity of $\omega$ at $r$. By Egoroff's theorem there exists a subset $C$ of $A$ such that $\omega(C)>1-\delta$ and the functions $x \rightarrow x t_{n}: X \rightarrow X$ converge uniformly on $C$ to the constant function $x \rightarrow b: X \rightarrow X$.

Now let $V$ be a neighborhood of $b$. Then there exist neighborhoods $W$ of $s$ and $U$ of $b$ such that $U \subset V$ and $x t \in V s(x \in U, t \in W)$.

There exists $N$ such that $y t_{n} \in U, S_{n} \in W$ and $r_{n} \in U_{C}(y \in C$, $n \geq N$ ) where $U_{C}$ is the neighborhood of $r$ guaranteed by 3.5.

Since $C \cap C r_{N} \neq \varnothing, C \cap C r_{N}^{-1} \neq \varnothing$. Let $x_{N} \in C$ with $x_{N} r_{N} \in$ $C$. Then on the one hand $x_{N} r_{N} t_{N} \in U$ and on the other $x_{N} r_{N} t_{N}=$ $x_{N} t_{N} s_{N} \in U s_{N} \subset V s$. Thus $V \cap V s \neq \varnothing$. The proof is completed.

The aim of the next few results is to determine some conditions which will ensure regularity. 
3.7. Proposition. Let $t \in \operatorname{supp} \mu$ be such that the map $s \rightarrow \omega\left(A s^{-1}\right)$ : $T \rightarrow \mathbb{R}$ is continuous at $t$ for all Borel subsets $A$ of $X$. Then (i) there exists $\delta>0$ such that $\omega(C)>1-\delta$ implies $\omega\left(C t^{-1}\right)>\delta$ for all Borel subsets $C$ of $X$ and (ii) $\omega$ is regular at $t$.

Proof. Assume (i) false. Then there exist a sequence of Borel sets $\left(C_{n}\right)$ with $\omega\left(C_{n}\right)>1-2^{-(n+1)}$ and $\omega\left(C_{n} t\right)<2^{-(n+1)}, n=1, \ldots$ Set $K_{n}=\bigcup\left\{C_{i} \mid 1 \leq i \leq n\right\}, K=\bigcup\left\{K_{n} \mid n=1, \ldots\right\}$. Then

$$
\omega\left(K_{n} t\right) \leq \sum_{i=1}^{n} \omega\left(C_{i} t\right) \leq \sum_{i=1}^{\infty} 2^{-(i+1)}=\frac{1}{2} \text { for all } n,
$$

whence $\omega(K t)=\lim _{n \rightarrow \infty} \omega\left(K_{n} t\right) \leq \frac{1}{2}$. However $\omega(K) \geq \omega\left(K_{n}\right) \geq$ $\omega\left(C_{n}\right) \geq 1-2^{-(n+1)}$ implies that $\omega(K)=1$ and this contradicts 3.3.

(ii) Let $\delta$ be as in (i) and $C$ a closed subset with $\omega(C)>1-\delta$. Then $\omega\left(C t^{-1}\right)>\delta$ whence there exists a neighborhood $U_{C}$ of $t$ such that $\omega\left(C s^{-1}\right)>\delta\left(s \in U_{C}\right)$. Hence $C \cap C s^{-1} \neq \varnothing \neq C \cap C s\left(s \in U_{C}\right)$.

3.8. Definition [A, pg. 21]. The measure $\mu$ is spread out (etalée) if either of the following equivalent conditions is satisfied: (i) there exists an integer $n$ such that $\mu^{n}$ is not singular with respect to a Haar measure $m$ on $T$, (ii) the set $\Sigma_{\mu}$ is not empty where $\Sigma_{\mu}$ is the set of elements $t$ of $T$ for which there exists an integer $p$ such that $\mu^{p}$ dominates a multiple of $m$ on some neighborhood of $t$.

3.9. Remark. In Proposition I.6 of [A] Azencott shows that every bounded measurable $\mu$-harmonic function is continuous if $\mu$ is spread out. Thus in this case $\omega$ is regular at $t$ for every $t$ in the support of $\mu$.

3.10. Review. We now deduce other results similar to 3.6 but before doing so we review some measure theory. Let $X$ be a locally compact space, $\operatorname{Meas}(X)$ the set of measures on $C, \mathscr{K}(X)=(f \in C(X): \operatorname{supp} f$ compact $\}$, and $\alpha, \beta \in \operatorname{Meas}(X)$. Then $\|\alpha\|=\sup \{\langle f, \alpha\rangle \mid f \in K(X)$, $\|f\|=1\}, \alpha \leq \beta$ if $\langle f, \alpha\rangle \leq\langle f, \beta\rangle(f \in \mathscr{K}(X)$ with $0 \leq f)$ and $\alpha \wedge \beta=$ infimum of $\alpha$ and $\beta$.

When $X$ is compact, $\mathscr{M}(X)=\{\alpha \in \operatorname{Meas}(X):\|\alpha\|=1\}$.

The proof of the following proposition is straightforward and will be omitted.

3.11. Proposition. Let $(X, T)$ be a flow, $\gamma \in \mathscr{M}(X)$ and $\alpha, \beta \in$ $\mathscr{M}(\beta T)$. Then (i) $\alpha \geq \beta$ implies $\gamma \alpha \geq \gamma \beta$, (ii) $\gamma \alpha \wedge \gamma \beta \geq \gamma(\alpha \wedge \beta)$, (iii) $\|\gamma \alpha \wedge \gamma \beta\| \geq\|\gamma\|\|\alpha \wedge \beta\|=\|\alpha \wedge \beta\|$. 
3.12. Proposition. Let $\lambda, \eta, \rho \in \mathscr{M}(X)$ and $\left(\lambda_{\alpha}\right),\left(\eta_{\alpha}\right)$ be nets in $\mathscr{M}(X)$ such that $\lambda_{\alpha} \rightarrow \lambda, \eta_{\alpha} \rightarrow \eta$ and $\lambda_{\alpha} \wedge \eta_{\alpha} \rightarrow \rho$. Then $\lambda \wedge \eta \geq \rho$.

Proof. Let $0 \leq f \in C(X)$ and $\varepsilon>0$. Since

$$
\lambda \wedge \eta(f)=\inf \{\lambda(g)+\eta(h) \mid 0 \leq g, h \in C(X) ; g+h=f\}
$$

[B1] there exist $g, h \in C(X)$ with $0 \leq g, h ; g+h=f$ and $\lambda \wedge \eta(f)>$ $\lambda(g)+\eta(h)-\varepsilon$.

There exists $\alpha$ such that $\lambda_{\alpha} \wedge \eta_{\alpha}(h)>\rho(h)-\varepsilon, \lambda_{\alpha}(g)<\lambda(g)+\varepsilon$, and $\eta_{\alpha}(h)<\eta(h)+\varepsilon$, whence $\lambda \wedge \eta(f)>\lambda_{\alpha}(g)+\eta_{\alpha}(h)-3 \varepsilon \geq \lambda_{\alpha} \wedge \eta_{\alpha}(h)-3 \varepsilon$ $>\rho(h)-4 \varepsilon$.

3.13. Corollary. Let $\lambda, \eta \in \mathscr{M}(X)$. Then the map $(t, s) \rightarrow\|\lambda t \Lambda \eta s\|:$ $T \times T \rightarrow \mathbb{R}$ is upper semicontinuous.

Proof. This follows from 3.12 and the fact that $\|\lambda\|=\lambda(1)(\lambda \in$ $\mathscr{M}(X))$.

3.14. Theorem. Let $r \in T$ and $\left(t_{\alpha} \mid \alpha \in I\right)$ a net in $T$ with $\omega t_{\alpha} \rightarrow$ $x \in X$. Then $x r=x$ if either (i) there exists $s \in T$ with $\omega_{s} t_{\alpha} \rightarrow x$ and $t_{\alpha}^{-1} s t_{\alpha} \rightarrow r$ or (ii) there exist $r_{\alpha} \in T(\alpha \in I)$ and $0<C \in \mathbb{R}$ such that $r_{\alpha} \rightarrow r \in T$ and $\left\|\omega \wedge \omega t_{\alpha} r_{\alpha} t_{\alpha}^{-1}\right\|>C$ for all $\alpha$.

Proof. (i) This follows immediately from the continuity of the map $(\eta, t) \rightarrow \eta t: \mathscr{M}(X) \times T \rightarrow \mathscr{M}(X)$.

(ii) Since $\omega t_{\alpha} \rightarrow \delta_{x}, \omega t_{\alpha} r_{\alpha} \rightarrow \delta_{x} r$ and $\left\|\omega t_{\alpha} \wedge \omega t_{\alpha} r_{\alpha}\right\|=$ $\left\|\omega \wedge \omega t_{\alpha} r_{\alpha} t_{\alpha}^{-1}\right\|>C$ for all $\alpha,\left\|\delta_{x} \wedge \delta_{x} r\right\|>C$ by 3.13. This implies that $x=x r$.

3.15. REMARKs. 1. Let $X$ be metrizable and $\left(t_{n}\right)$ a sequence in $T$ with $\omega t_{n} \rightarrow x \in X$. Then there exists $A \subset X$ such that $\omega(A)=$ 1 and $y t_{n} \rightarrow x(y \in A)$. Now let $s \in T$ with $\omega\left(A s^{-1}\right) \neq 0$ and $t_{n}^{-1} s t_{n} \rightarrow r \in T$. Then there exists $y \in A \cap A s^{-1}$ whence $y t_{n} \rightarrow x$ and $y s t_{n} \rightarrow x$. Consequently $x r=\left(\lim y t_{n}\right) \lim \left(t_{n}^{-1} s t_{n}\right)=\lim y s t_{n}=x$. Thus when $X$ is metrizable (i) can be strengthened. Moreover in this case $\mu\left\{s \mid \omega\left(A s^{-1}\right)=1\right\}=1$.

2. Let $t, s \in T$. Then one way to ensure that $\|\omega t \wedge \omega s\|>C$ is to find measures $\lambda, \rho \in \mathscr{M}(\beta T)$ such that $\omega \lambda=\omega=\omega \rho$ and $\|\lambda t \wedge \rho s\|>C$, for by $3.11\|\omega t \lambda \omega s\|=\|\omega \lambda t \wedge \omega \rho s\| \geq\|\lambda t \wedge \rho s\|>C$.

The following result due to Furstenberg [F2] (see above [A, p. 76]) is thus relevant for our considerations. 
3.16. Lemma. Let $t_{1}, t_{2} \in \Sigma_{\mu}$. Then there exist $U$ open, $\varepsilon>0$ and $\theta \in \operatorname{cnv}\left\{\mu^{n} \mid n=1, \ldots\right\}$ such that $t_{1}, t_{2} \in U$ and $r, s \in T$ with $U r \cap U s \neq \varnothing$ implies $\left\|\theta r \wedge \theta_{s}\right\| \geq \varepsilon$.

Proof. There exist open relatively compact neighborhoods $V_{1}$ and $V_{2}$ of $t_{1}$ and $t_{2}$ respectively, integers $p, q$ and $a_{1}, a_{2}$ positive elements of $\mathbb{R}$ such that $\chi_{\bar{V}_{1}} \mu^{p} \geq a_{1} \chi_{\bar{V}_{1}} m$ and $\chi_{\bar{v}_{2}} \mu^{q} \geq a_{2} \chi_{\bar{v}_{2}} m$.

Now set $U=V_{1} \cup V_{2}$ and $a=\min \left(a_{1}, a_{2}\right)$. Then

$$
\frac{a}{2} \chi_{U} m \leq \chi_{v}\left(\left(\mu^{p}+\mu^{q}\right) / 2\right) \text {. }
$$

Consequently by [A, Lemma IV.3 p. 76] there exists $\varepsilon>0$ such that $r, s \in T$ with $U r \cap U s \neq \varnothing$ implies $\left\|\eta^{2} r \wedge \eta^{2} s\right\| \geq \varepsilon$ where $\eta=\left(\mu^{p}+\mu^{q}\right) / 2$ whence $U, \varepsilon$, and $\theta=\eta^{2}=\frac{1}{4}\left(\mu^{2 p}+\mu^{p+q}+\mu^{2 q}\right)$ satisfy the requirements of 3.5 .

3.17. Standing notation. For the remainder of this section we again adopt the notation of 2.5 , with $\mu \in \mathscr{M}(T)$ and $\nu \in \mathscr{L}(\mu)$. (The subscript $\nu$ will be dropped.)

In addition $(X, T)$ will now denote an arbitrary factor of $(B, T)$ and $\omega$ the measure induced by the one on $B$.

3.18. Theorem. Let $p \in S$, $\left(t_{\alpha}\right)$, $\left(s_{\alpha}\right)$ nets in $T$ such that $t_{\alpha} \rightarrow p$, $s_{\alpha} \rightarrow s \in T$, and $t_{\alpha} s_{\alpha} t_{\alpha}^{-1} \rightarrow t \in \Sigma_{\mu}^{-1} \Sigma_{\mu}$. Then (i) $\omega p \in X$ and (ii) $(\omega p) s=(\omega p)$.

Proof. Statement (i) follows from 2.17.

To see (ii) let $t=t_{1}^{-1} t_{2}$ with $t_{1}, t_{2} \in \Sigma_{\mu}$ and $U, \theta, \varepsilon$ as in 3.16. Since $t_{1} t=t_{2}, U t \cap U \neq \varnothing$ whence there exists $\alpha_{0}$ such that $U t_{\alpha} s_{\alpha} t_{\alpha}^{-1} \cap U \neq \varnothing$ $\left(\alpha \geq \alpha_{0}\right)$. Hence $\left\|\theta \wedge \theta t_{\alpha} s_{\alpha} t_{\alpha}^{-1}\right\| \geq \varepsilon>0\left(\alpha \geq \alpha_{0}\right)$ and (ii) follows from 3.15 and 3.14 .

3.19. Corollary. Let $h \in T$ be such that given $p \in S$ there exist $a$ net $\left(t_{\alpha}\right)$ in $T$ and $t \in \Sigma_{\mu}^{-1} \Sigma_{\mu}$ with $t_{\alpha} h t_{\alpha}^{-1} \rightarrow t$ and $t_{\alpha} \rightarrow p$. Then (i) $x h=x(x \in B)$ and (ii) $h f=f\left(f \in \mathscr{H}_{\mu}\right)$.

Proof. Statement (i) follows from 3.18 with $X=B$ and the fact that $X=\{\omega p \mid p \in S\}$.

The second part of 3.19 follows immediately from the first.

3.20. Remarks. 1. An element $h$ in $T$ with $h f=f\left(f \in \mathscr{H}_{\mu}\right)$ is what Azencott [A, p. 7] calls a $\mu$-period. Corollary 3.19 is intimately related to Theorem IV.1 of $[\mathbf{A}]$. 
Another result along these lines is the following which relies on 3.6 rather than 3.18 .

3.21. Theorem. Let $T$ be second countable, $\mu$ spread out and $h \in T$ be such that given $p \in S$ there exists a net $\left(t_{\alpha}\right)$ in $T$ and $t \in \operatorname{supp} \mu$ with $t_{\alpha} \rightarrow p$ and $t_{\alpha} h t_{\alpha}^{-1} \rightarrow t$. Then $h f=f\left(f \in \mathscr{H}_{\mu}\right)$.

Proof. It suffices to show that $x h=x(x \in B)$ and since $T$ is second countable we may restrict ourselves to a metric factor $X$ of $B$.

Now let $x \in X$. Then there exists a sequence $\left(t_{n}\right)$ in $T$ such that $\omega t_{n} \rightarrow x$ and $t_{n} h t_{n}^{-1} \rightarrow r$ for some $r \in \operatorname{supp} \mu$. Set $s_{n}=h, r_{n}=t_{n} h t_{n}^{-1}$, $n=1, \ldots$ Then $t_{n} s_{n}=r_{n} t_{n}, s_{n} \rightarrow h, r_{n} \rightarrow r$ and by $3.7 \omega$ is regular at $r$. Since $\omega t_{n} \rightarrow x$, there exists a subset $A$ of $X$ with $\omega(A)=1$ and $y t_{n} \rightarrow x(y \in A)$. Consequently $x h=x$ by 3.6 .

4.0. Actions of subgroups on the Poisson space. In this section we study the action of a subgroup $K$ of $T$ on the space $B_{\nu}$ when the only $K$-invariant harmonic funtions are the constants. The idea is to reduce the study of the Poisson flow $(B, T)$ to the flow $(B, K)$.

4.1. Notation. In this section $\mu$ will denote an element of $\mathscr{M}(T)$ with supp $\mu=T$ and $\nu$ a fixed element of $\mathscr{L}(\mu)$. The notation of 1.3 will be used except that the subscript " $\nu$ " will be omitted.

Again $(X, T)$ will denote a homomorphic image of $B$ and $\omega \in \mathscr{M}(X)$ with $\omega \nu=\omega$.

Let $H \subset T$. Then $\mathscr{R}_{H}$ will denote the right uniformly continuous and $\mathscr{B}_{H}$ the bounded Borel functions $f$ on $T$ such that $h f=f(h \in H)$, and $\mathscr{B}_{\mu}$ the bounded Borel functions $g$ on $T$ such that $g \mu=g$.

4.2. Proposition. Let $K$ be a subgroup of $T$ and $U$ a non-vacuous open subset of $B$. Then $\omega(U K)=1$ if either (i) $\mathscr{B}_{K} \cap \mathscr{B}_{\mu}=\mathbb{R}$, (ii) $\mathscr{R}_{K} \cap \mathscr{B}_{\mu}=\mathbb{R}$ and $\mu$ spread out, or (iii) $\mathscr{R}_{K} \cap \mathscr{B}_{\mu}=\mathbb{R}$ and $K$ compact.

Proof. (i) Set $C=U K$ and assume that $\omega(C)<1$. Let $f \in C(X)$ with $0 \leq f \leq \chi_{C},\|f\|=1$. Then $f \omega(t) \leq \chi_{C} \omega(t)=\omega\left(C t^{-1}\right)=\Omega(C)$ since $\chi_{C} \omega \in \mathscr{B}_{K} \cap \mathscr{B}_{\mu}$. Thus $\|f \omega\| \leq \omega(C)<1$ and this contradicts the fact that $g \rightarrow g \omega: C(X) \rightarrow \mathscr{H}_{\mu}$ is an isometry.

(ii) In this case $\chi_{C} \omega \in \mathscr{R}_{K} \cap \mathscr{B}_{\mu}$ and the proof proceeds as in (i). 
(iii) Again let $f$ and $C$ be as in (i) and let

$$
g(x)=\sup \{f(x k) \mid k \in K\} \text {. }
$$

Then $g$ is a $K$-invariant continuous function on $X$ with $\|g\|=1$ and $g \leq \chi_{C}$, whence $g \omega \in \mathscr{R}_{K} \cap \mathscr{B}_{\mu}$ with $\|g \omega\|<1$. The proof is completed.

4.3. Remarks. 1. Since supp $\omega=X, \overline{U K}=X$ under any of the conditions of 4.2 .

2. The group $T$ satisfies (i) of 4.2 whence $(B, T)$ is topologically transitive i.e. $\overline{U T}=B$ for all non-vacuous open subsets of $B$.

3. Let $X$ be a metric fator of $B, \omega_{X}$ the measure induced on $X$ by $\omega$, $\left(U_{i} \mid i=1, \ldots\right)$ a countable base for the topology on $X$ and $K$ as in 4.2. Then $E=\bigcap_{i=1}^{\infty} U_{i} K$ is a residual subset of $X$ such that $\omega_{X}(E)=1$ and $\overline{x K}=X(x \in E)$.

4. Let $K$ be as in (iii) of 4.2. Then $x K=B(x \in B)$. (This follows from 4.2 and the fact that $B=\overline{U K}=\bar{U} K$ for every neighborhood $U$ of $x$.)

4.4. Proposition. Let $(X, T)$ be a metric factor of $(B, T), K$ a compact second countable normal subgroup of $T$ with $\mathscr{R}_{K} \cap \mathscr{H}_{\mu}=\mathbb{R}$, and $\mu$ spread out. Then $X$ is a point.

Proof. Let $\eta$ be the measure on $X$ induced by $\omega$ and $\left(t_{n}\right)$ a sequence in $T$ such that $x t_{n} \rightarrow z \in X(x \in A)$ with $\eta(A)=1$.

Let $k \in K$ and set $r_{n}=t_{n} k t_{n}^{-1}$. Then $r_{n} \in K$ for all $n$ and we may assume that $r_{n} \rightarrow r \in K$.

Now $r_{n} t_{n}=t_{n} k$ for all $n$, and $\eta$ is regular at $r$ by 3.9. Consequently $z k=z$ by 3.6. Thus $X=z K=z$ by 4.3.

4.5. Proposition. Let $(X, T)$ be a metric factor of $(B, T), K$ a compact normal subgroup of $T$ with $\mathscr{R}_{K} \cap \mathscr{H}_{\mu}=\mathbb{R}$, and $\left.\operatorname{ad}_{K} r \mid r \in T\right\}$ a relatively compact subset of the group of automorphisms of $K$. Then $X$ is a point. (Here $\operatorname{ad}_{K} t: K \rightarrow K$ is the map such that $\left(\operatorname{ad}_{K} t\right)(k)=t k t^{-1}$ $(k \in K)$.)

Proof. Let $a \in X$ and $\varphi: K \rightarrow X$ the map such that $\varphi(k)=a k$ $(k \in T)$. Then $\varphi$ is an open surjective map (4.3).

Now let $A,\left(t_{n}\right)$, and $b$ be as in the proof of 3.6, and $\alpha$ an automorphism of $K$ adherent to the sequence $\operatorname{ad}_{K} t_{n}^{-1}$. Then $\varphi^{-1}(A)$ is a dense subset of $K$ and $k \in \varphi^{-1}(A)$ implies that $b=\lim a k t_{n}=$ $\lim a t_{n} \operatorname{ad}_{K} t_{n}^{-1}(k)=b \alpha(k)$. Since $\varphi^{-1}(A)$ is dense, this implies that $b=b h(h \in K)$. The proof is completed. 
The following is surely well known. We include a proof for lack of a reference.

4.6. Lemma. Let $K$ be a compact analytic Lie group. Then the identity component $\operatorname{aut}_{0}(K)$ of the group of automorphisms, aut $(K)$ of $K$ is compact.

Proof. It follows from $[\mathbf{H}]$ that $K$ is isomorphic to $\left(\mathbb{R}^{n} \times G\right) / H$ where $G$ is a simply connected compact semi-simple group and $H$ is a discrete group containing $\mathbb{Z}^{n} \times e$. Hence aut $(K)=\operatorname{aut}\left(\mathbb{R}^{n} \times G, H\right)=$ $\{u \in \operatorname{aut}(\mathbb{R} \times G) \mid u(H)=H\}[\mathbf{B} 2]$.

Let $u \in \operatorname{aut}_{0}(K), \pi, \kappa$ the canonical maps of $\mathbb{R}^{n} \times G$ onto $\mathbb{R}^{n}$ and $G$ respectively. Since $\mathbb{R}^{n}$ has no non-trivial compact subgroups the homomorphism $g \rightarrow \pi(u(0, g)): G \rightarrow \mathbb{R}^{n}$ must be the trivial one. Also $G$ semisimple implies that the homomorphism $r \rightarrow \kappa(u(r, e))$ is trivial.

Since $u$ is homotopic to the identity, $H$ is discrete and $u(H)=H$, $u(h)=h(h \in H)$. In particular $u(z, e)=(z, e)\left(z \in \mathbb{Z}^{n}\right)$ whence $u(r, e)=(r, e)\left(r \in \mathbb{R}^{n}\right)$. Hence $u(r, g)=u(r, e) \cdot u(0, g)=(r, e)(0, \bar{u}(g))$ $=(r, \bar{u}(g))$ where $\bar{u}$ is the automorphism of $G$ given by $g \rightarrow \kappa u(0, g)$. Thus $\operatorname{aut}_{0}(K)$ is isomorphic to $\operatorname{aut}_{0}(G)$ which is compact $[\mathbf{H}]$.

4.7. Proposition. Let $T$ be connected and $K$ a compact normal subgroup of $T$ such that $\mathscr{R}_{K} \cap \mathscr{H}_{\mu}=\mathbb{R}$. Then $\mathscr{H}_{\mu}=\mathbb{R}$.

Proof. It suffices of course to show that the Poisson flow $(B, T)$ is trivial. Since $(B, T)$ is topologically transitive, we need only show that $T$ acts trivially on $B$.

Let $U$ be a neighborhood of the identity of $T$. Then there exists a compact normal subgroup $L$ of $T$ such that $L \subset U$ and $T / L$ is a Lie group [M.Z.]. Then $T / L$ acts on $B / L$ which is then an image of the Poisson flow associated with the measure induced by $\mu$ on $T / L$. Thus we may assume that $T$ is a Lie group.

It follows readily from 2 of 4.3 that $B$ is connected. Also $K$ acts transitively on $B$ by 3 of 4.3 . Since $K / K_{0}$ is finite, this implies that the component of the identity, $K_{0}$ of $K$ acts transitively on $K$. Hence $\mathscr{R}_{K_{0}} \cap \mathscr{H}_{\mu}=\mathbb{R}$.

Finally $\operatorname{ad}_{K_{0}}(T)$ is contained in aut $0\left(K_{0}\right)$ and so is relatively compact by 4.6. Proposition 4.7 now follows from 4.5. 
5.0. The solvable group case. In this section we show that when $T$ is a solvable second countable connected group and $M$ is spread out with $\mu=\mu^{-1}, \mathscr{H}_{\mu}=\mathbb{R}$ (see [B.R.]).

Let $\varphi: T \rightarrow T$ be such that $\varphi(t)=t^{-1}(t \in T)$ and $f \in \mathscr{R}$. Then in general $f \circ \varphi \notin \mathscr{R}$. Thus in order to take advantage of the assumption $\mu=\mu \circ \varphi$ we must work with a larger algebra than $\mathscr{R}$, one on which $\mu$ is defined and which is invariant under $\varphi$. In this section we use $C_{b}(T)$, the algebra of bounded continuous functions on $T$.

The group $T$ acts on the Gelfand space $\left|C_{b}(T)\right|$ of $C_{b}(T)$, but the map $(x, t) \rightarrow x t:\left|C_{b}(T)\right| \times(T, \mathscr{T}) \rightarrow\left|C_{b}(T)\right|$ is in general not continuous. However this is unnecessary for our purposes.

5.1. Standing notation. In this section $T$ will denote a connected Lie group, $\mu$ a spread out probability measure on $T$ with $\mu=\mu^{-1}$ (i.e. $\mu(A)=\mu\left(A^{-1}\right)$ for all Borel subsets of $\left.T\right)$ and $\nu \in \overline{\operatorname{cnv}}\left(\mu^{n} \mid n=1, \ldots\right)$ with $\nu \in \mathscr{L}(\mu)$ and $\kappa_{\nu}\left(S_{\nu}\right)=B_{\nu}$. (Such exists by 2.17.).

Again the notation of $\S 1$ will be used with the subscript $\nu$ omitted.

Since $T$ is second countable, the flow $(B, T)$ is the inverse limit of its metric factors. In this section $(X, T)$ will denote an arbitrary one of these, $\kappa_{X}$ or simply $\kappa$ the canonical map of $S$ onto $X$ and $\omega_{X}$ or simply $\omega$, the measure on $X$ induced by $\nu$.

Finally $\varphi: \beta T \rightarrow \beta T$ will denote the continuous extension to $\beta T$ of the map $t \rightarrow t^{-1}: T \rightarrow T \subset \beta T$, and $C_{b}(T)=\left\{f \in C(\beta T):\left.f\right|_{T}\right.$ is continuous $\}$. We shall also denote the affine extension of $\varphi$ to $\mathscr{M}(\beta T)$ by $\varphi$.

5.2. Remarks. 1. $C_{b}(T)$ is a uniformly closed $T$-invariant subalgebra of $C(\beta T)$ which may be identified with the algebra of bounded continuous funtions on $T$.

2. $\mathscr{R} \subset C_{b}(T)$.

3. $f \circ \varphi \in C_{b}(T)$ and $\mu(f)=\mu(f \cdot \varphi)\left(f \in C_{b}(T)\right)$.

5.3. Lemma. (i) There exists $\gamma \in \overline{\mathrm{cnv}}\left\{\mu^{n} \mid n=1, \ldots\right\} \subset \mathscr{M}\left(C_{b}(T)\right)$ such that $\gamma^{2}=\gamma=\varphi(\gamma)$ and $\gamma \mu=\gamma$. (ii) $\langle f, \gamma\rangle=\langle f, \nu\rangle(f \in \mathscr{R})$.

Proof. Let $K=\overline{\operatorname{cnv}}\left\{\mu^{n} \mid n=1, \ldots\right\} \subset \mathscr{M}\left(C_{b}(T)\right), r$ the restriction mapping of $\mathscr{M}\left(C_{b}(T)\right)$ onto $\mathscr{M}(\mathscr{R})$ and $L=r(K)$. Then clearly $L=$ $\overline{\operatorname{cnv}}\left\{\mu^{n} \mid n=1, \ldots\right\} \subset \mathscr{M}(\mathscr{R})$ whence $\nu \in L$.

Now $\alpha \mu \in K(\alpha \in K)$ whence $\alpha \beta \in K(\alpha, \beta \in K)$. Consequently $C=r^{-1}(\nu) \cap K$ is a non-empty convex compact subset of $K$ such that $C^{2} \subset C$ and $C \mu \subset C$. This in turn implies that $A=\{\alpha \in C \mid \alpha \mu=\alpha\}$ 
is a non-empty compact convex subset of $C$ with $A^{2}=A$. Hence there exists $\gamma^{2}=\gamma \in A$.

To see that $\varphi(\gamma)=\gamma$ observe that $\varphi\left(\delta_{t} \delta_{s}\right)=\varphi(t s)=s^{-1} t^{-1}=$ $\varphi\left(\delta_{s}\right) \varphi\left(\delta_{t}\right)(s, t \in T)$. Since $\varphi$ is linear this implies that $\varphi(\alpha \beta)=$ $\varphi(\beta) \varphi(\alpha)\left(\alpha, \beta \in \operatorname{cnv}(T) \subset \mathscr{M}\left(C_{b}(T)\right)\right)$ and by continuity of $\varphi$ and the maps $\rho \rightarrow \rho \alpha: \mathscr{M}\left(C_{b}\right) \rightarrow \mathscr{M}\left(C_{b}\right)$ and $\rho \rightarrow \alpha \rho: \mathscr{M}\left(C_{b}\right) \rightarrow \mathscr{M}\left(C_{b}\right)$ $(\alpha \in \mathscr{M}(T))$ that $\varphi\left(\mu^{n}\right)=\mu^{n}$ for all $n$. Hence $\varphi(\gamma)=\gamma$. The proof is completed.

5.4. Standing notation. For the remainder of this section $K$ will denote a closed subgroup of $T$ with $\mathscr{R}_{K} \cap \mathscr{H}_{\mu}=\mathbb{R}$.

5.5. Lemma. Let $E=\{x \in X \mid \overline{x K}=X\}$. Then there exists a sequence $\left(t_{n}\right)$ in $T$ such that $\omega t_{n} \rightarrow x_{1} \in E$ and $\omega t_{n}^{-1} \rightarrow x_{2} \in E$.

Proof. Let $\gamma$ be as in 5.3, $\Sigma=\operatorname{supp} \gamma$, and $\eta$ the canonical map of $\Sigma$ onto $S=$ supp.

By 2 of $4.3 \omega(E)=1$ whence $\gamma(F)=1$ where $F=\eta^{-1}\left(\kappa^{-1}(E)\right)$.

Now let $\psi:\left|C_{b}(T)\right| \rightarrow\left|C_{b}(T)\right|$ be the map induced by $\varphi$. Since $\varphi(\gamma)=\gamma, \psi(\Sigma)=\Sigma$ and $\gamma(\psi(F))=1$. Hence there exists $p \in \beta T$ such that $\pi(p) \in F$ and $\pi(\varphi(p))=\psi(\pi(p)) \in F$, and so $\omega p, \omega \varphi(p) \in E$ (where $\pi:\left(\beta(T, e) \rightarrow\left(\left|C_{b}(T)\right|, e\right)\right.$ is the natural map).

When $p$ is viewed as an ultra filter on $T, \varphi(p)=\left\{U^{-1} \mid U \in p\right\}$ and $\omega p=\lim _{p} \omega t, \omega \varphi(p)=\lim _{\varphi(p)} \omega t$.

Let $V_{n}\left(W_{n}\right)$ be a neighborhood base for $\omega p$ and $\omega \varphi(p)$ respectively and choose $U_{n} \in p$ such that $\omega t \in V_{n}$ and $\omega s \in W_{n}$ for all $n, t \in U_{n}$, $s \in U_{n}^{-1}$.

Finally choose $t_{n} \in U_{n} n=1, \ldots$ Then $t_{n}^{-1} \in U_{n}^{-1}$ so that $\omega t_{n} \rightarrow$ $\omega p, \omega t_{n}^{-1} \rightarrow \omega \varphi(p)$.

5.6. LemMA. Let $K$ be normal, $H$ the identity component of the center of $K$, and assume $H_{0} \neq\{e\}$. Then there exists $h \in H$ with $h \neq e$ and $x h=x(x \in X)$.

Proof. Assume 5.6 false and let $\left(t_{n}\right)$ be a sequence in $T$ such that $\omega t_{n} \rightarrow x_{1}, \omega t_{n}^{-1} \rightarrow x_{2}$ and $\overline{x_{1} K}=X=\overline{x_{2} K}$.

Since $K \triangleleft T$, ad $t_{n}(H)=H=\operatorname{ad} t_{n}^{-1}(H)$. Let $\mathscr{N}$ be the set of open connected neighborhoods of the identity in $H, V, W \in \mathscr{N}$, and $A(V, W)=\left\{n \mid t_{n}^{-1} V t_{n} \cap W^{\prime} \neq \varnothing\right\}$.

We claim that $n \in A(V, W)$ implies that $t_{n}^{-1} V t_{n} \cap(\bar{W} \backslash W) \neq \varnothing$. To see this assume $t_{n}^{-1} V t_{n} \cap(\bar{W} \backslash W)=\varnothing$. Then $t_{n}^{-1} V t_{n} \cap \bar{W}=t_{n}^{-1} V t_{n} \cap W$ 
is both open and closed in $t_{n}^{-1} V t_{n}$. Since $t_{n}^{-1} V t_{n}$ is connected and $e \in t_{n}^{-1} V t_{n} \cap W, t_{n}^{-1} V t_{n}=t_{n}^{-1} V t_{n} \cap W \subset W$ a contradiction.

Now fix $W \in \mathscr{N}$. We claim that there exist $V \in \mathscr{N}$ and an integer $N$ with $t_{n}^{-1} V t_{n} \subset W$ for all $n \geq N$. Otherwise by the preceding remark there would be a subsequence $\left(t_{n_{t}}\right)$ of $\left(t_{n}\right)$ and a sequence $\left(r_{i}\right) \rightarrow e$ with $s_{i}=t_{n_{i}}^{-1} r_{i} t_{n_{i}} \in \bar{W} \backslash W$ for all $i$. We may assume $s_{i} \rightarrow s \in \bar{W} \backslash W$. Then $r_{i} t_{n_{l}}=t_{n_{t}} s_{i}, r_{i} \rightarrow e, s_{i} \rightarrow s \neq e$ whence by $3.6 x_{1} s=x_{1}$. (Recall that $\mu$ spread out with supp $\mu=T$ implies that every element of $T$ is regular with respect to $\omega$.) Then $x_{1} k=x_{1} s k=x_{1} k s(k \in K)$ and so $x s=x(x \in X)$, a fact which contradicts our original assumption that 5.6 was false.

A similar argument replacing the sequence $\left(t_{n}\right)$ by the sequence $\left(t_{n}^{-1}\right)$ shows that given $W \in \mathcal{N}$ there exist an integer $N$ and $V \in \mathcal{N}$ with $t_{n} V t_{n}^{-1} \subset W$ for all $n \geq N$.

Finally choose $V, W$ as above with $\bar{W}$ compact and let $e \neq r \in V$. Then we may assume $s_{n}=t_{n} r t_{n}^{-1} \rightarrow s \in \bar{W}$. Again by $3.6 x_{1} s=x_{1}$ and so $x s=x(x \in X)$. Moreover the preceding paragraphs show that $s \neq e$, which again contradicts our original assumption. The proof is completed.

5.7. Proposition. Let $K$ be normal and $H$ the identity component of the center of $K$. Then $H$ acts trivially on $X$.

Proof. Let $G=\{t \in T \mid x t=x(x \in X)\}$. Then $G$ is a closed normal subgroup of $T$.

Let $\pi: T \rightarrow T / G$ be the canonical map. Then $\mu \pi^{-1}$ is a spread out measure on $T / G$ with $\left(\mu \pi^{-1}\right)^{-1}=\mu \pi^{-1}, T / G$ acts on $X$ and $(X, T / G)$ is a metric factor of the Poisson flow associated with $\mu \pi^{-1}$. Consequently the results of this section apply with $T, K, \mu$ replaced by $T / G, \pi(K), \mu \pi^{-1}$ respectively.

Now $\pi(H)$ is contained in the identity component of the center of $\pi(K)$ so that the latter would not be trivial if $H$ were not contained in $G$. But this would contradict 5.6.

5.8. TheOREM. Let $T$ be a connected solvable Lie group, $\rho$ a spread out measure on $T$ such that $\rho=\rho^{-1}$ and the subgroup generated by the support of $\rho$ equals $T$. Then $\mathscr{H}_{\rho}=\mathbb{R}$.

Proof. Set $\mu=\sum_{\rho=1}^{\infty} 2^{-n} \rho$. Then $\mu=\mu^{-1}$, supp $\mu=T$, and $\mathscr{H}_{\rho} \subset$ $\mathscr{H}_{\mu}$. Thus it suffices to prove that $\mathscr{H}_{\mu}=\mathbb{R}$ where $\mu$ is as before. 
Theorem 5.8 is clear if $\operatorname{dim} T=0$. Let $n>1$ and assume 5.8 for all positive integers less than $n$. Set $N=[T, T]$. Then $N$ is a closed normal subgroup of $T$ with $\operatorname{dim} N<n$.

If $\operatorname{dim} N=0, T$ is abelian and the result is well known in that case.

Now assume $\operatorname{dim} N>0$. Then $\operatorname{dim} T / N<n$ and so $\mathscr{H}_{\mu \pi^{-1}}=\mathbb{R}$ where $\pi: T \rightarrow T / N$ is the canonical map. Since every element of $\mathscr{R}_{N} \cap \mathscr{H}_{\mu}$ induces an element of $\mathscr{H}_{\mu \pi^{-1}}, \mathscr{R}_{N} \cap \mathscr{H}_{\mu}=\mathbb{R}$.

Let $H$ be the identity component of the center of the nilpotent group $N$. Then $\operatorname{dim} H \geq 1$ and by $5.7 H$ acts trivially on every metric factor of $B$ and since $(B, T)$ is the inverse limit of its metric factors, $H$ acts trivially on $B$. Then $(B, T)=(B, T / H)$ is a factor of the Poisson flow of the measure induced by $\mu$ on $T / H$ and the latter is trivial since $\operatorname{dim} T / H<n$. The proof is completed.

6.0. The semi-simple case. In this section we use the methods developed in this paper to prove Furstenberg's result [F2]: let $T=$ $K A N$ be the Iwasawa decomposition of the semi-simple Lie group $T$ with finite center and no compact factors; then $K$ acts transitively on the Poisson space of $\mu$ for every supported spread out measure $\mu$ on $T$.

6.1. Notation and review. In this section we retain the notation of 5.1 with the assumption that $T$ is an analytic semi-simple Lie group with finite center and no compact factors.

Let $\mathbf{t}$ be the Lie algebra of $T, \mathbf{t}=\mathbf{k}+\mathbf{p}$ a Cartan decomposition of $\mathbf{t}, \mathbf{a}$ a maximal abelian subspace of $\mathbf{p}$ and $\Delta$ the roots of the pair $(\mathbf{t}, \mathbf{a})$. Order $\Delta$ and let $\Delta^{+}$be the positive elements of $\Delta$.

For $\lambda \in \Delta$ set $\mathbf{t}^{\lambda}=\{Y \in \mathbf{t} \mid[H, Y]=\lambda(Y), H \in \mathbf{a}\}$ and define the Lie subalgebras $\mathbf{n}^{ \pm}$by $\mathbf{n}^{ \pm}=\sum_{ \pm \lambda \in \Delta^{+}} \mathbf{t}^{\lambda}$ and let $K, A, N^{ \pm}$be the analytic subgroups of $T$ corresponding to $\mathbf{k}, \mathbf{a}, \mathbf{n}^{ \pm}$respectively.

Let $\mathbf{a}^{+}=\left\{H \in \mathbf{a} \mid \lambda(H)>0, \lambda \in \Delta^{+}\right\}$and $A^{+}$the corresponding analytic subgroup of $T$.

Then $K$ is compact, $K A^{+} K=T=K A N^{+}=K A N^{-}$.

6.2. LEMMA. There exist sequences $\left(k_{n}\right)$ in $K$ and $\left(a_{n}\right)$ in $A^{+}$such that $\omega k_{n} a_{n} \rightarrow x_{0} \in X$, with $\overline{x_{0} T}=X$.

Proof. Let $\left(t_{n}\right)$ be a sequence in $T$ with $\omega t_{n} \rightarrow x \in X$ and with $\overline{x T}=X$, such exist by 4.3. Write $t_{n}=k_{n} a_{n} l_{n}$ with $\left(k_{n}\right),\left(l_{n}\right) \subset K$ and $\left(a_{n}\right) \subset A^{+}$. We may assume $l_{n} \rightarrow l \in K$ and $\omega k_{n} a_{n} \rightarrow \rho \in \mathscr{M}(X)$. 
Then $\omega t_{n}=\omega k_{n} a_{n} l_{n} \rightarrow \rho l$, whence $\lim \omega k_{n} a_{n}=\rho=x l^{-1} \in X$. Set $x_{0}=x l^{-1}$. Then $\overline{x_{0} T}=\overline{x l^{-1} T}=\overline{x T}=X$.

6.3. Standing notation. For the rest of this section $\left(a_{n}\right),\left(k_{n}\right)$ will denote fixed sequences in $A^{+}$and $K$ respectively such that $\omega k_{n} a_{n} \rightarrow$ $x_{0} \in X$ with $\overline{x_{0} T}=X$. We shall also assume that $k_{n} \rightarrow k \in K$.

Our aim is to show that $x_{0} K=X$.

6.4. Lemma. Let $a \in A$. Then $x_{0} a=x_{0}$.

Proof. Since $A$ is abelian, $k_{n} a_{n} a=k_{n} a a_{n}=k_{n} a k_{n}^{-1} k_{n} a_{n}$. Now $k_{n} a k_{n}^{-1} \rightarrow k a k^{-1}$ whence $x_{0} a=x_{0}$ (apply 3.6 to the sequences $\left(t_{n}\right)=$ $\left.\left(k_{n} a_{n}\right),\left(s_{n}\right)=(a),\left(r_{n}\right)=\left(k_{n} a k_{n}^{-1}\right)\right)$.

The proof of the next lemma is standard and will be omitted.

6.5. Lemma. Let $Y, H \in \mathbf{t}$ with $[H, Y]=\lambda Y$ for some $\lambda \in \mathbb{R}$. Then $(\exp H)(\exp Y)(\exp (-H))=\exp \left(e^{\lambda} Y\right)$.

6.6. LemMA. Let $-\lambda \in \Delta^{+}, Y \in \mathbf{t}^{\lambda}$, and $t=\exp Y$.

Then $x_{0} t=x_{0}$.

Proof. Let $a_{n}=\exp H_{n}$ with $H_{n} \in \mathbf{a}^{+}$for all $n$. Then $a_{n} t a_{n}^{-1}=$ $\exp \left(e^{\lambda\left(H_{n}\right)} Y\right)$ and since $\lambda\left(H_{n}\right)<0$ we may assume that the sequence $e^{\lambda\left(H_{n}\right)}$ converges. Hence the sequence $r_{n}=a_{n} t a_{n}^{-1}$ converges to $r \in T$. Finally $k_{n} a_{n} t=k_{n} r_{n} a_{n}=k_{n} r_{n} k_{n}^{-1} k_{n} a_{n}$ and so again $x_{0} t=x_{0}$ by 3.6 since $k_{n} r_{n} k_{n}^{-1} \rightarrow k r k^{-1} \in T$.

6.7. LemmA. The group $K$ acts transitively on $X$; indeed $x_{0} K=X$.

Proof. Let $H=\left\{t \in T \mid x_{0} t=x_{0}\right\}$. Then $H$ is a closed subgroup of $T$ which contains $A$ by 6.4. By $6.6 \exp Y \in H Y$, for all $Y \in \mathbf{t}^{\lambda}$ and $-\lambda \in \Delta^{+}$. Hence the Lie algebra of $H$ contains that of $N^{-}$and so $N^{-} \subset H$.

Finally $X=\overline{x_{0} T}=\overline{x_{0} N^{-} A K}=\overline{x_{0} K}=x_{0} K$.

6. THEOREM (Furstenberg [F2]). (i) The group $k$ acts transitively on $B$, (ii) $\mathscr{R}_{K} \cap \mathscr{H}_{\mu}=\mathbb{R}$.

Proof. Let $b \in B$ and assume $c \notin b K$. Then $c K \cap b K=\varnothing$ and there exists $f \in C(B)$ with $f(x)=0(x \in b K)$ and $f(x)=1(x \in c K)$. 
Set $R=\{(x, y) \mid f(x t)=f(y t)(t \in T)\}$. Then $R$ is a closed invariant equivalence relation on $B$ and $(X, T)=(B / R, T)$ is a metric factor of $B$ since $T$ is 2 nd countable.

Let $\pi: B \rightarrow X$ be the canonical map. Then $f(c) \neq f(b k)$ shows that $\pi(c) \neq \pi(b) k(k \in K)$. Thus $K$ does not act transitively on $X$, a fact which contradicts 6.7 .

(ii) This follows immediately from (i).

\section{REFERENCES}

[A] R. Azencott, Espace de Poisson des Groupes Localement Compact, Berlin, Springer-Verlag, 1970 (Lecture notes in mathematics 148).

[B1] N. Bourbaki, Elements de Mathematique XIII Integration, Hermann, Paris 1965.

[B2] __ Elements de Mathematique, Groupes et algebres de Lie, Chapitres 4, 5, 6, Hermann, Paris 1968.

[B.R.] L. Berge and A. Rauge, Fonctions harmoniques sur les groupes moyenable, Comptes Rendu 278 Serie A (1974), 1287.

[D] D. Dokken, $\mu$-Harmonic functions on locally compact groups, (to appear in Journal d'Analyse Mathematique).

[E] R. Ellis, Lectures on Topological Dynamics, W. A. Benjamin, New York 1969.

[F1] H. Furstenberg, Random walks and discrete subgroups of Lie groups, Adv. Prob. and related topics (1).

[F2] _ A Poisson formula for semi-simple Lie groups, Annals of Math., 77 (1963), 335-383.

[G] S. Glasner, Proximal Flows, Springer-Verlag, Berlin (Lecture notes in mathematics 517).

[H] G. Hochschild, The structure of Lie groups, Holden-Day, San Francisco, 1965.

[M.Z.] D. Montgomery and L. Zippin, Topological Transformation Groups, New York, Interscience Publishers (1955).

Received March 2, 1988 and in revised form November 8, 1988. Research partially supported by NSF grant 8701857 .

College of St. Thomas

St. PAUL, MN 55105

AND

UNIVERSITY OF MINNESOTA

MINNEAPOLIS, MN 55455 



\section{PACIFIC JOURNAL OF MATHEMATICS EDITORS}

\author{
V. S. VARADARAJAN \\ (Managing Editor) \\ University of California \\ Los Angeles, CA 90024-1555-05 \\ Herbert Clemens \\ University of Utah \\ Salt Lake City, UT 84112 \\ ThOMAS ENRIGHT \\ University of California, San Diego \\ La Jolla, CA 92093
}

R. FINN

Stanford University

Stanford, CA 94305

HeRmann FlaschKa

University of Arizona

Tucson, AZ 85721

VAUGHAN F. R. JONES

University of California

Berkeley, CA 94720

Steven KerckHofF

Stanford University

Stanford, CA 94305
RoBION KIRBY

University of California

Berkeley, CA 94720

C. C. MOORE

University of California

Berkeley, CA 94720

Harold Stark

University of California, San Diego

La Jolla, CA 92093

\section{ASSOCIATE EDITORS}

\begin{tabular}{|c|c|c|c|c|}
\hline R. ARENS & $\begin{array}{l}\text { E. F. BECKENBACH } \\
(1906-1982)\end{array}$ & H. NeumanN & F. Wolf & K. YoshidA \\
\hline \multicolumn{5}{|c|}{ SUPPORTING INSTITUTIONS } \\
\hline \multicolumn{2}{|c|}{ UNIVERSITY OF ARIZONA } & \multicolumn{3}{|c|}{ UNIVERSITY OF OREGON } \\
\hline \multirow{2}{*}{\multicolumn{2}{|c|}{$\begin{array}{l}\text { UNIVERSITY OF BRITISH COLUMBIA } \\
\text { CALIFORNIA INSTITUTE OF TECHNOLOGY }\end{array}$}} & \multicolumn{3}{|c|}{ UNIVERSITY OF SOUTHERN CALIFORNIA } \\
\hline & & \multicolumn{3}{|c|}{ STANFORD UNIVERSITY } \\
\hline \multicolumn{2}{|c|}{ UNIVERSITY OF CALIFORNIA } & \multicolumn{3}{|c|}{ UNIVERSITY OF HAWAII } \\
\hline \multicolumn{2}{|c|}{ MONTANA STATE UNIVERSITY } & \multicolumn{3}{|c|}{ UNIVERSITY OF TOKYO } \\
\hline \multicolumn{2}{|c|}{ UNIVERSITY OF NEVADA, RENO } & \multicolumn{3}{|c|}{ UNIVERSITY OF UTAH } \\
\hline \multicolumn{2}{|c|}{ NEW MEXICO STATE UNIVERSITY } & \multicolumn{3}{|c|}{ WASHINGTON STATE UNIVERSITY } \\
\hline OREGON STA & UNIVERSITY & \multicolumn{3}{|c|}{ UNIVERSITY OF WASHINGTON } \\
\hline
\end{tabular}




\section{Pacific Journal of Mathematics}

\section{Vol. 141, No. $1 \quad$ November, 1990}

Yusuf Abu-Muhanna and Abdallah Khalil Lyzzaik, The boundary

behaviour of harmonic univalent maps $\ldots \ldots \ldots \ldots \ldots \ldots \ldots \ldots \ldots \ldots$

Lawrence Jay Corwin, Allen Moy and Paul J. Sally, Jr., Degrees and

formal degrees for division algebras and $\mathrm{GL}_{n}$ over a $p$-adic field $\ldots \ldots \ldots 21$

Ulrich Dierkes and Gerhard Huisken, The $n$-dimensional analogue of the

catenary: existence and nonexistence $\ldots \ldots \ldots \ldots \ldots \ldots \ldots \ldots 47$

Peter Gerard Dodds, C. B. Huijsmans and Bernardus de Pagter,

Characterizations of conditional expectation-type operators $\ldots \ldots \ldots \ldots 55$

Douglas Dokken and Robert Ellis, The Poisson flow associated with a

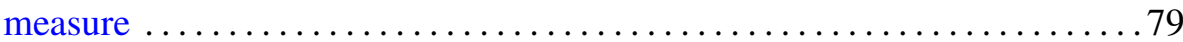

Larry J. Santoni, Horrocks' question for monomially graded modules . . . . 105

Zbigniew Slodkowski, Pseudoconvex classes of functions. II. Affine

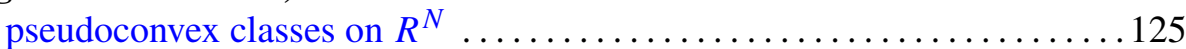

Dean Ellis Smith, On the Cohen-Macaulay property in commutative algebra

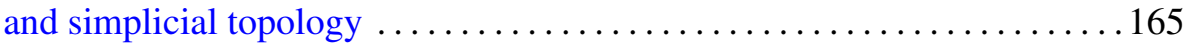

Michał Szurek and Jaroslaw Wisniewski, Fano bundles over $P^{3}$ and $Q_{3} \ldots 197$ 